\title{
On the Use of Singular Vectors for the Flexibility-Based Damage Detection under the Assumption of Unknown Structural Masses
}

\author{
Said Quqa (D), Luca Landi, and Pier Paolo Diotallevi \\ Department DICAM, University of Bologna, Viale Risorgimento 2, 40136 Bologna, Italy \\ Correspondence should be addressed to Said Quqa; said.quqa2@unibo.it
}

Received 26 October 2017; Revised 24 December 2017; Accepted 18 January 2018; Published 28 February 2018

Academic Editor: Luca Zanotti Fragonara

Copyright (C) 2018 Said Quqa et al. This is an open access article distributed under the Creative Commons Attribution License, which permits unrestricted use, distribution, and reproduction in any medium, provided the original work is properly cited.

\begin{abstract}
The main purpose of this work is to investigate the usability of easily obtainable parameters instead of the modal traditional ones, in the context of a flexibility-based damage detection procedure, under the assumption of unknown structural masses. To this aim, a comparison is made between two different approaches: the first involves the calculation of the flexibility matrix by using traditional modal parameters, such as natural frequencies and modal vectors, normalized to unitary values, while the second involves the use of singular vectors, obtained through a simple matrix factorization. The modal parameters and the singular vectors necessary for the implementation of the damage detection procedure are evaluated through two different techniques: the Eigensystem Realization Algorithm and a wavelet-based procedure, for which a variant is proposed by introducing the energy reassignment concept into the original algorithm. Through the latter approach, in particular, it is possible to obtain a high number of singular vectors even in the case of reduced availability of sensors. The study is performed under the assumption of nonstationary excitation, in order to achieve general results, and the effectiveness of the procedures is evaluated through simulated tests regarding different structural schemes.
\end{abstract}

\section{Introduction}

Seismic risk, meant as an estimate of the damage expected in a given time interval, is determined by the combination of three factors: seismic hazard, measured on the frequency and the energy of earthquakes registered in a certain area, vulnerability (i.e., the proneness of a building to manifest damage in occurrence of a seismic event), and the exposure, evaluated on the number of assets exposed to risk. In view of the seismic risk reduction, it is possible to operate only on the vulnerability factor, by adopting appropriate measures in the design phase for the newly built structures or by monitoring the existing ones in order to ensure the necessary safety interventions.

In most of the practical cases, the safety of a civil building is traditionally assessed on the basis of qualitative direct observations, driven by experience, often accompanied by invasive techniques [1]. Together with the need of detecting more accurate data related to the structural operational conditions and thanks to the recent improvements of sensing technology, several techniques have been developed in the
Vibration-Based Structural Health Monitoring (SHM) scenario, through the processing of data collected by suitable sensors (generally accelerometers), arranged on the monitored structure $[2,3]$.

One of the main purposes of SHM is the qualitative identification of a possible damage on the operative structure and its localization $[1,4,5]$. The damage generally entails a reduction of structural performances, modeled as a stiffness decrease. It is also assumed that the damage directly affects natural frequencies and modal shapes but does not involve any change in masses. Flexibility-based approaches are among the most used methods for damage detection and entail the computation of the flexibility matrix at two different time instants, in order to evaluate the variation between these matrices, which can be interpreted as a variation of the structural characteristics. In order to build the flexibility matrix, natural frequencies and modal shapes are needed [6], which can be evaluated through dynamic identification techniques.

In literature, two main methods for conducting modal identification are widely described: Experimental Modal 


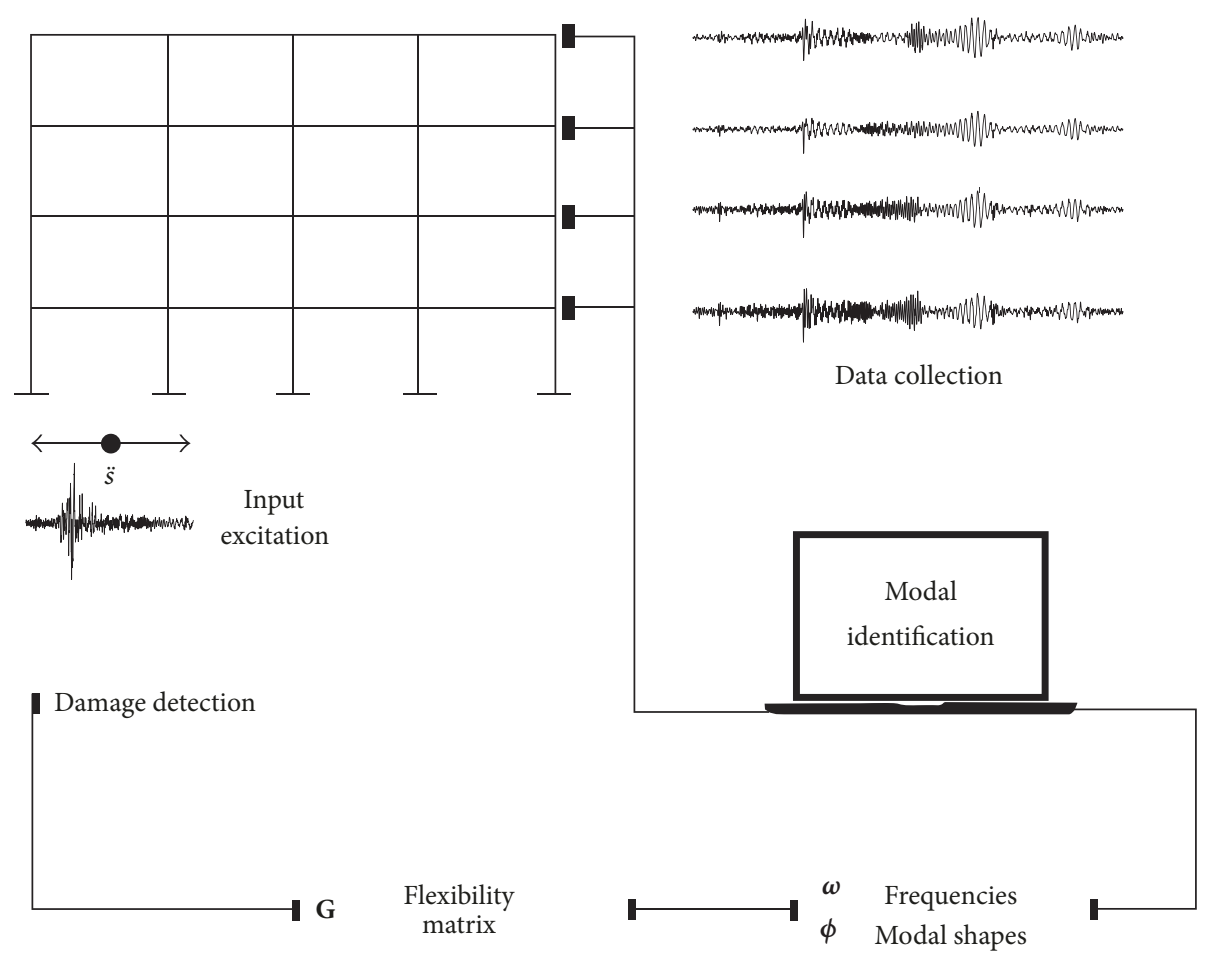

FIGURE 1: Synthetic scheme of a flexibility-based damage detection procedure.

Analysis (EMA), in which the identification procedure is performed starting from input and output data [7], and Operational Modal Analysis (OMA), in which only the output data is used $[8,9]$. The difficulty of implementing the EMA for large-scale structures and its high economic burden have shifted the interest of the most recent studies towards outputonly methods, in which the input is given by the natural excitation (i.e., wind, traffic, and minor seismic phenomena) [10]. Most of the algorithms used in OMA are based on the assumption of modeling the input signal as white noise with stationary characteristics. Owing to the partial enforceability of the aforementioned assumptions (e.g., sudden changes in wind direction and speed) [11], some recent researches refer to techniques based on the assumption of nonstationary inputs. In this context, new tools have been developed, such as the Wigner-Ville distribution and the wavelet transform, which allow the joint time-frequency domain representation of the signal [11-18].

The most recently implemented procedures in these research fields go towards the increase in performance, preferring fast and computationally less expensive methods. In the context of flexibility-based damage detection, the main objective of this paper is to propose and evaluate the effectiveness of an approach that uses a mixing matrix, obtained through Singular Value Decomposition (SVD) [19], compared to the traditional approach that involves the modal matrix (Figure 1), under the assumption of unknown masses. It is known from the literature that a fast estimation of modal vectors from the dynamic structural responses can be obtained through Blind Source Separation (BSS) techniques [9], which provide a mixing matrix with properties different from those of the real modal matrix. In particular, the SVD is one of the simplest techniques used for BSS, and its use would allow simplifying the whole damage detection procedure.

Since the SVD would provide a number of singular vectors (i.e., the columns of the mixing matrix) equal to the number of recorded dynamic structural responses, a Wavelet Scalogram and Singular Value Decomposition-based technique (WS-SVD) $[20,21]$ or a transformed version (WSTSVD) [11, 22-25] could be applied for signal sparsification in case of reduced availability of sensors, in order to allow the extraction of modal responses, which can be used for the estimation of a larger set of singular vectors. These procedures also allow the estimation of natural frequencies, even in the case of nonstationary excitation. Since a correct estimate of these parameters is of the utmost importance for the flexibility-based damage detection, a variant of the original WS-TSVD-based procedure is proposed, aimed at further improving the obtained results. In particular, two steps are introduced within the original workflow: the first entails an energy reassignment process, widely applied in the field of signal analysis [18, 26-29], and the second consists of an energy-based filtering procedure.

The combination of the Reassigned WS-TSVD-based method (RWS-TSVD) for signal sparsification and the SVDbased approach for the computation of the flexibility matrix would allow fast recognizing structural damage by approximate calculations, without a large number of sensors and the knowledge of structural masses, only by using the acceleration time histories recorded at different points of the analyzed structure. In this way, contrary to other methods that provide specific tests to mass-normalize the modal matrix [30, 31], it 
is possible to estimate the damage without interrupting the ordinary use of the analyzed structure. After a first quick test, the procedure could be reperformed by moving the sensors, in order to investigate more accurately the damage entity and location.

The paper is organized as follows: in Section 2 the SVD and the analogies with modal superposition are recalled; in Section 3 the wavelet-based sparsification and identification procedure is briefly described, focusing on the highlights of the proposed variant; in Section 4 the flexibility-based damage detection is discussed, with particular attention to the use of singular vectors as damage sensitive parameters and to the approximations caused by the lack of mass matrix. In Section 5 the described procedures are applied on discrete and continuous systems, by simulated tests performed through a finite element software.

In order to study the effectiveness of the examined procedures, several comparisons are reported in Section 5. Firstly, the estimated error on the natural frequencies obtained through the RWS-TSVD-based method is evaluated. Subsequently, a comparison is made between the frequencies obtained by the RWS-TSVD-based procedure, the traditional WS-TSVD-based algorithm, and the consolidated Eigensystem Realization Algorithm (ERA) method [32]. After evaluating errors on natural frequencies, those on the flexibility matrices are estimated. In particular, the matrix computed through natural frequencies and modal vectors, obtained by using the ERA, will be compared to the matrix computed through singular vectors and natural frequencies, estimated by the RWS-TSVD-based method. Finally, the errors on the damage estimates are studied, comparing the results obtained by using the two different flexibility matrices described above.

\section{Singular Value Decomposition and Modal Superposition}

In this section, we briefly recall the basic theory of Singular Value Decomposition and the analogies with the modal superposition problem. From now on, with the notation $\mathbf{A} \epsilon$ $\mathbb{R}^{a \times b}$ we mean that $\mathbf{A}$ is a real matrix with $a$ rows and $b$ columns, while with the notation $\mathbf{a} \in \mathbb{R}^{a}$ we mean that a is a real column vector with $a$ terms. Also, we call $n$ the number of Degrees of Freedom (DOFs) of the structure and $m$ the number of sensors arranged on it. For the discrete systems, since every DOF is associated with an independent movement and with recorded time histories we intend the acceleration relative to an independent movement, it is assumed that control points can be chosen within the set of DOFs (and $m \leq n$ ).

Considering $\mathbf{H} \in \mathbb{R}^{m \times k}$ the matrix whose rows represent the acceleration time histories $\mathbf{h}_{j}$ (each containing $k$ time samples) collected at the $j$ th control point of the structure, every row can be expressed as a linear combination of all the $n$ structural responses, represented as columns in the matrix $\mathbf{S}=\left[\mathbf{s}_{1}, \mathbf{s}_{2}, \ldots, \mathbf{s}_{n}\right] \in \mathbb{R}^{k \times n}:$

$$
\mathbf{H}=\Sigma \overline{\boldsymbol{\Phi}} \mathbf{S}^{\mathrm{T}},
$$

where $\overline{\boldsymbol{\Phi}}=\left[\bar{\phi}_{1}, \bar{\phi}_{2}, \ldots, \bar{\phi}_{n}\right] \in \mathbb{R}^{n \times n}$ is the modal massnormalized matrix (i.e., the norm of each modal vector is equal to $\alpha_{i}=\left(\boldsymbol{\phi}_{i}^{\mathrm{T}} \mathbf{M} \boldsymbol{\phi}_{i}\right)^{-1 / 2}$, where $\mathbf{M}$ is the mass matrix of the structure and $\left.\phi_{i}=\bar{\phi}_{i} /\left\|\bar{\phi}_{i}\right\|\right)$ and $\Sigma \in \mathbb{R}^{m \times n}$ is a selection matrix whose rows are each equal to zero vectors, except for the $i$ th element equal to 1 , which corresponds to the position of the $j$ th control point. Considering the case in which the number of sensors is equal to the number of DOFs $(m \equiv n)$, the matrix $\boldsymbol{\Sigma}$ becomes an identity matrix of order $n$, and the problem can be described as

$$
\mathbf{H}=\overline{\boldsymbol{\Phi}} \mathbf{S}^{\mathrm{T}}=\sum_{i=1}^{n} \alpha_{i} \boldsymbol{\phi}_{i} \mathbf{s}_{i}^{\mathrm{T}} .
$$

Since the aim of identification process is that of estimating modal parameters by analyzing registered time histories, neither $\boldsymbol{S}$ structural responses nor the modal matrix $\overline{\boldsymbol{\Phi}}$ are known. The modal superposition problem described above lies in the class of unmixing problems, on which a Blind Source Separation (BSS) technique can be applied with the aim of estimating the mixing matrix $\overline{\boldsymbol{\Phi}}$. If the number of mixed recordings $m$ is equal to the number of sources $n$ (columns of $\mathbf{S}$ ), the problem is determined and can be solved directly. Otherwise, if $m<n$, that is, the most common case in the modal identification problems, $\bar{\Phi}$ can be estimated after sparsifying the registrations, that is, changing the domain of the registered mixes in order to bring out a number of independent parameters characterizing the sources, equal to or greater than $n$.

Focusing on the determined problem $(m \equiv n)$, the BSS can be carried out by means of a SVD of the recordings matrix. In particular, a real matrix $\mathbf{H} \in \mathbb{R}^{m \times k}$ can be factorized as

$$
\mathbf{H}=\Psi \mathbf{Z X}^{\mathrm{T}} \text {, }
$$

where $\Psi=\left[\psi_{1}, \psi_{2}, \ldots, \psi_{m}\right]$ is a $m \times m$ matrix such that $\boldsymbol{\Psi} \boldsymbol{\Psi}^{\mathrm{T}}=\boldsymbol{\Psi}^{\mathrm{T}} \boldsymbol{\Psi}=\mathbf{I}$, where $\mathbf{I}$ is the identity matrix, whose columns $\psi_{j}$ (namely, the left singular vectors) are a set of orthonormal eigenvectors of $\mathbf{H H}^{\mathrm{T}}$. Similarly, $\mathbf{X}=\left[\boldsymbol{\chi}_{1}, \boldsymbol{\chi}_{2}, \ldots\right.$, $\left.\chi_{k}\right]$ is a $k \times k$ matrix composed of the right singular vectors $\chi_{l}$ (with $l=1, \ldots, k$ ), which are a set of orthonormal eigenvectors of $\mathbf{H}^{\mathrm{T}} \mathbf{H}$, and $\mathbf{Z}$ is the $m \times k$ diagonal matrix of singular values, which are the square roots of nonzero eigenvalues of both $\mathbf{H H}^{\mathrm{T}}$ and $\mathbf{H}^{\mathrm{T}} \mathbf{H}$. The $\mathbf{H}$ matrix can thus be expressed as a sum of contributions given by the outer product of the $j$ th left $\psi_{j}$ and right $\chi_{j}$ singular vectors, each weighted by the $j$ th nonzero element $z_{j}$ of the matrix $\mathbf{Z}$ :

$$
\mathbf{H}=\sum_{j=1}^{m} z_{j} \psi_{j} \boldsymbol{\chi}_{j}^{\mathrm{T}}
$$

Starting from the latter representation, since the modulus of $z_{j}$ decreases when the $j$ index increases, it is simple to note the analogies between the modal matrix, whose columns consist in ordered modal vectors, and the matrix $\Psi$, whose columns represent the sets of coefficients that, multiplied by the right singular vectors, better approximate the matrix 

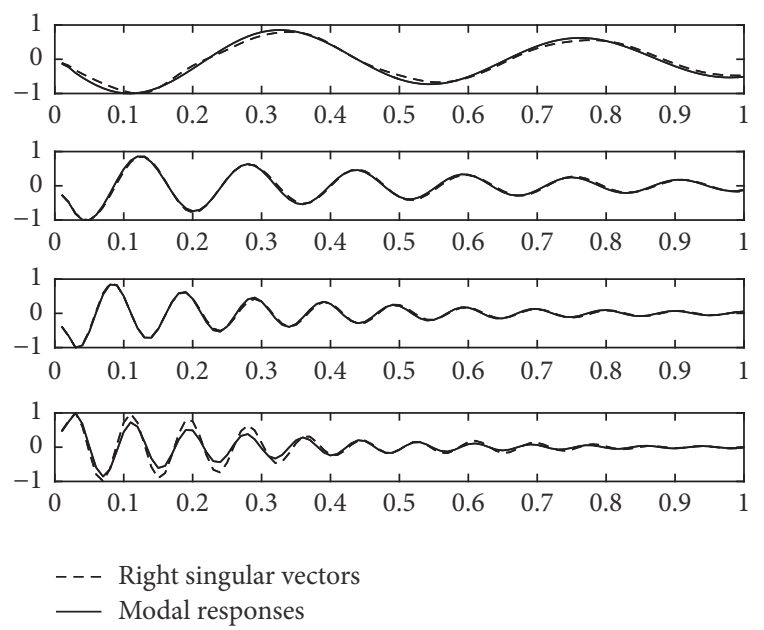

FIGURE 2: Comparison between right singular vectors, extracted by means of SVD, and modal responses of a 4-DOF share-type frame in free vibration, with reference to the example at Section 5.1.3. For an effective comparison, the vectors are both normalized to the maximum value and only the first 100 terms are plotted.

H. In fact, a structural response signal can be effectively approximated by taking into account a number of vibration modes proportional to the desired precision level.

It is noted that the matrix $\Psi$ does not correspond exactly to the modal matrix, and thus the right singular vectors are different from the modal responses (Figure 2). In particular, $\Psi$ is composed of orthonormal vectors, that is, $\psi_{j}^{\mathrm{T}} \psi_{j}=1$, while $\overline{\boldsymbol{\Phi}}$ is composed of vectors such that $\bar{\phi}_{i}^{\mathrm{T}} \mathbf{M} \overline{\boldsymbol{\phi}}_{i}=1$ [33].

\section{Modal Identification}

The main purpose of dynamic identification is to determine modal parameters, represented by natural frequencies, damping ratios, and modal shapes, from dynamic recordings. In case the exciting input is nonstationary, assuming the analyzed system as linear, the structural response will also have time-varying characteristics [6]. For this reason, in order to obtain significant results, it is essential to conduct analyses in the time-frequency domain. Indeed, the vibration modes are easier to detect when the input has frequency values similar to the structural natural ones. If the input acceleration is characterized by a narrow band of frequencies, which varies over time, it is likely that not all modes can be identified simultaneously, but only at certain time intervals.

In this section we briefly describe the RWS-TSVD-based technique for signal sparsification and modal identification. The procedure is composed of three steps: the sparsification of the collected time histories (TH), the features detection, and the modal identification (Figure 3).

3.1. Signal Sparsification and Features Detection. In the first step the collected data is processed by continuous wavelet transform, in order to compute a scalogram, whose content can be interpreted as a signal energy density distribution [13]. Specifically, the analysis is carried out by selecting the
Morlet wavelet as mother function, which best suits harmonic signals, since the scale parameter is characterized by a unique relationship with the signal frequency obtainable by Fourier analysis [14].

In order to improve the scalograms readability and to reduce the interference caused by mutual energy between different signal components, an energy reassignment operation is performed. This technique involves assigning the calculated energy levels to the weighted centroid of the analysis windows rather than to their geometric center [18, 26-29]. In this way the energy peaks are more homogeneous in the different scales and have a lesser spread in the time-frequency plane.

Following the computation of scalograms, the areas of interest to extract the modal parameters should be evaluated. This phase consists of features detection, which can be done by decomposition of the two-dimensional distribution into vectors representing the energy density of the analyzed signal [11]. For this purpose, recent studies that use the SVD [20, 21] and the TSVD (whose bases are obtained by rotating the bases of the traditional method) [11,22-25] have been developed.

In the feature detection phase, the energy peaks associated with vibration modes are selected. It often happens that computational modes are also identified, which do not refer to the dynamic behavior of the system. In order to minimize user intervention in the identification procedure, a filter has been introduced into the algorithm to eliminate computational modes. In particular, the Root Mean Square (RMS) energy has been computed for each wavelet coefficients vector, and a threshold has been set to eliminate those with an energy value lower than the $10 \%$ of the highest RMS value.

As the RWS-TSVD-based algorithm detects narrow areas in terms of frequency, the corresponding wavelet coefficients can be interpreted as single modal responses and then processed as SDOF responses for the identification of modal parameters.

3.2. Extraction of Modal Parameters. Following the selection of the modes of interest, natural frequencies can be estimated by Fast Fourier Transform (FFT) of the wavelet coefficients extracted from each energy peak. It is noted that FFT does not consider the time aspect of the analyzed recordings, but each wavelet component is characterized by a narrow frequency band, making it possible to estimate natural frequencies without merging vibration modes. For the same reason, also the damping ratio can be computed for each wavelet component as for SDOF signals. Damping ratio can be considered as a further damage detection parameter [34] but is of difficult interpretation for the sake of damage magnitude evaluation. Therefore, in the present paper, we only focus on natural frequencies and modal shapes to formulate considerations on the damage level.

Regarding the modal shapes, in the case of large number of sensors (nearly determined problem), we investigate if the modal matrix can be assumed as the $\Psi$ matrix computed as explained in Section 2, considering the fact that the error increases with the $n / m$ ratio. Otherwise, in the case of strongly underdetermined problem $(m \ll n)$, the scalogram could result in a convenient sparse representation 


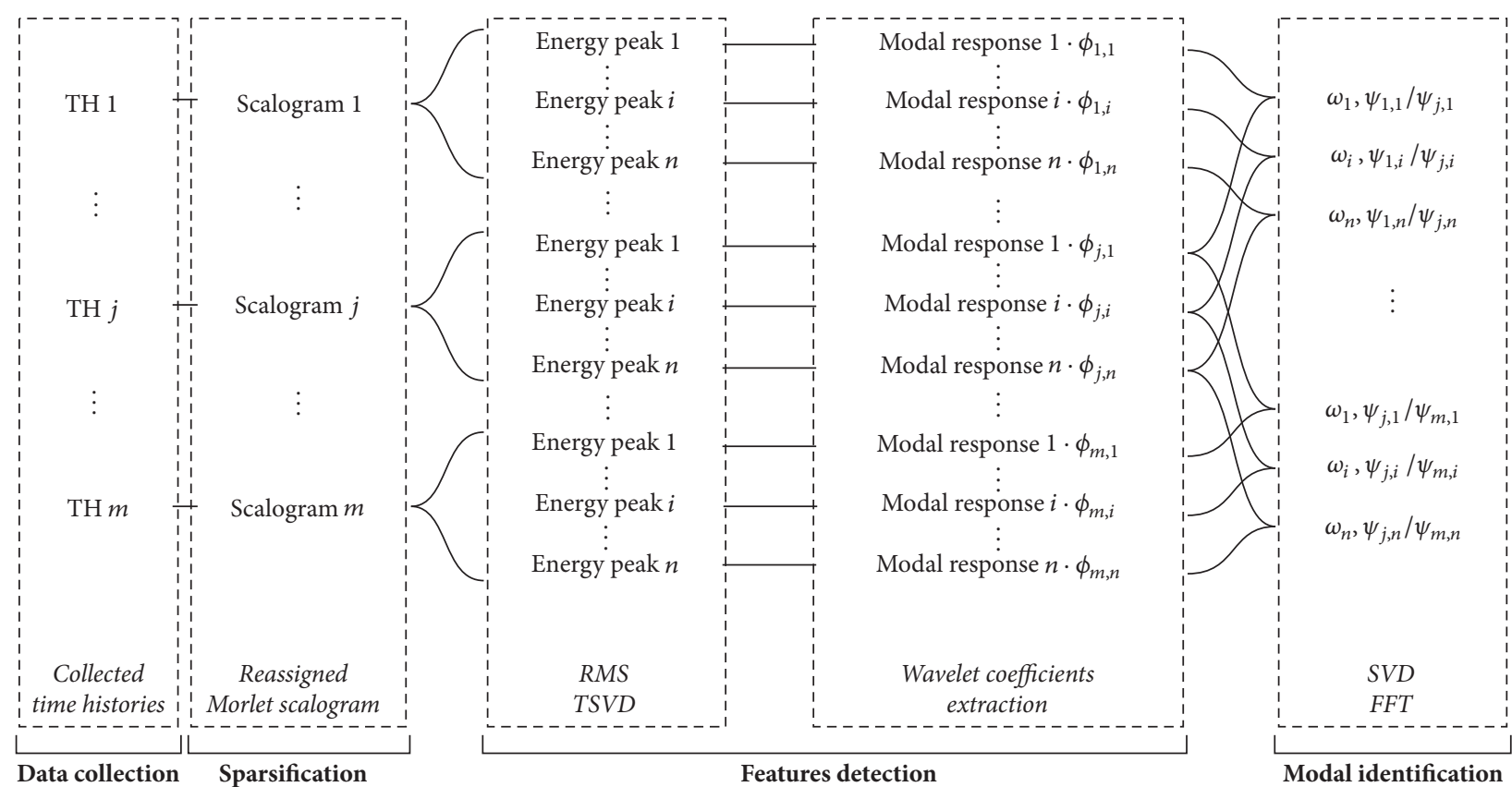

FIGURE 3: Workflow of the identification algorithm.

for each registered signal, since it highlights the energy peaks associated with single vibration modes. The BSS problem could thus be formulated by assuming the wavelet coefficients corresponding to each identified peak as a single source vector, multiplied by the $\phi_{j, i}$ element of modal matrix, where $j$ is the control point position index at which the registration is recorded and $i$ is the mode index that represents the peak identified in the $j$ th scalogram. This procedure allows extracting the $i$ th vector $\psi_{i} \in \mathbb{R}^{m}$ (whose $m$ elements are nearly proportional to the corresponding $m$ elements of the modal vector $\bar{\phi}_{i} \in \mathbb{R}^{n}$ associated with the frequency $\omega_{i}$ ) by computing the SVD of the matrix whose columns consist of wavelet coefficients, obtained at the same identified $i$ th natural frequency (energy peak), each corresponding to a different control point of the structure.

Considering $\mathbf{w}_{i, j} \in \mathbb{R}^{k}$ the wavelet coefficients vector computed at the circular frequency $\omega_{i}$ on the time history registered at the $j$ th control point, a SVD of the matrix $\mathbf{W}_{m \times k}^{(i)}=\left[\mathbf{w}_{i, 1}, \mathbf{w}_{i, 2}, \ldots, \mathbf{w}_{i, m}\right]^{\mathrm{T}}$ can be performed as

$$
\mathbf{W}^{(i)}=\Psi^{(i)} \mathbf{Z}^{(i)} \mathbf{X}^{(i) \mathrm{T}},
$$

where $\Psi^{(i)}$ is an $m \times m$ matrix, whose first column represents the vector $\psi_{i}$. In order to obtain a complete (or a larger) vector $\psi_{i}$, if the number of sensors is small compared to the number of DOFs $(m \ll n)$, repeated tests can be performed, after displacing the sensors and keeping at least one in the same position, to allow the normalization of the whole set of collected coefficients at the same value. Indeed, taking two elements $\psi_{a, i}$ and $\psi_{b, i}$ of the vector $\psi_{i}$, they are only representative of the ratio $\psi_{a, i} / \psi_{b, i}$, because of their normalization.
Several identification algorithms widely described in the literature allow estimating directly the modal matrix instead of a similar-meaning matrix, assuming that the matrix of structural masses is known. One of them is the ERA [32], originally implemented for impulse response signals, which allow the immediate description of the Hankel matrix, starting point of the algorithm. Thanks to the speed and accuracy of this method, several variants have been introduced in order to make it applicable to natural vibration signals. In this context, the Natural Excitation Technique (NExT) [35] and the Random Decrement technique (RD) $[36,37]$ were used to derive system impulse response functions from response time histories to stochastic input. The first mentioned technique uses signal correlation and is based on the assumption that the input is characterized by a stationary zero-mean white noise, with Gaussian distribution; the second technique instead refers to the average of different signal segments that have the same initial conditions.

If the mass matrix is unknown, the modal matrix obtained by ERA (or other methods) is not mass-normalized, and the $i$ th modal mass-normalized vector $\bar{\phi}_{i}$ is expressed as

$$
\bar{\phi}_{i}=\frac{\varphi_{i}}{\sqrt{\varphi_{i}^{\mathrm{T}} \mathbf{M} \varphi_{i}}},
$$

where $\varphi_{i}$ is the $i$ th nonnormalized modal vector. In order to obtain usable results for the sake of damage detection through flexibility-based methods, the structural flexibility matrix has to be computed. To this aim, without the knowledge of masses, a pursuable criterion is that of normalizing every modal estimated vector so that

$$
\boldsymbol{\phi}_{i}=\frac{\boldsymbol{\varphi}_{i}}{\sqrt{\boldsymbol{\varphi}_{i}^{\mathrm{T}} \mathbf{I} \boldsymbol{\varphi}_{i}}},
$$




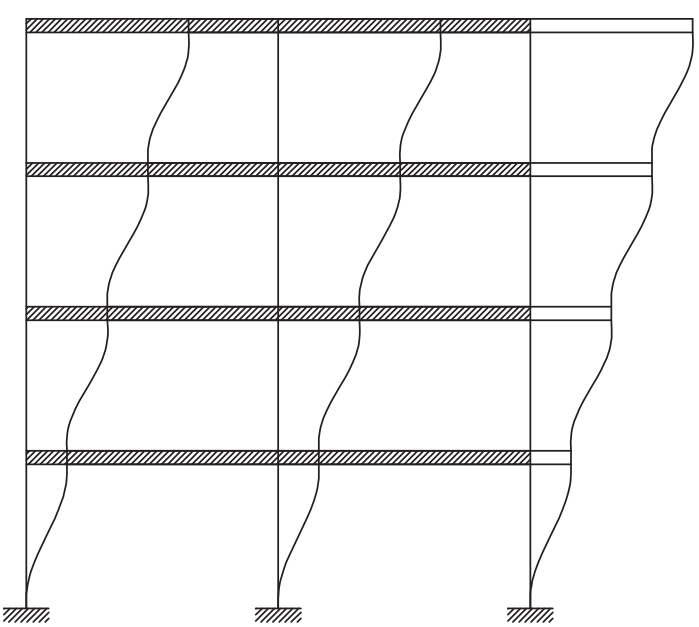

(a)

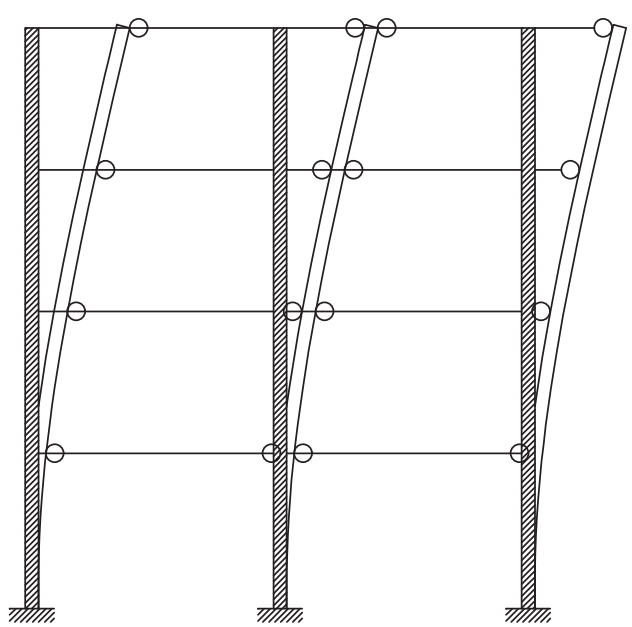

(b)

FIGURE 4: Modeling limit cases for a plane frame.

where $\mathbf{I}$ is the identity matrix and $\Phi=\left[\phi_{1}, \phi_{2}, \ldots, \phi_{n}\right]$ is the unitary-mass-normalized modal matrix. Because of its normalization, the flexibility matrix obtained from $\Phi$ is proportional to the flexibility matrix of a structure with a diagonal mass matrix such that $\mathbf{M}=\mu \mathbf{I}$, where $\mu$ is a constant value, equal to the mass associated with each DOF of the structure. It is easy to see that the more the masses are effectively equally distributed along the structure, the more precisely the described criterion is pursuable.

It is noted that $\Phi$ and $\Psi$ matrices are obtained by different procedures, and in particular, the matrix $\Psi$ is generally obtainable in an easier way, since it involves a simple matrix factorization. However, considering the similarities between relations (2) and (4) in addition to the analytical meaning of the two matrices, as explained above, $\Psi$ could be used in a flexibility-based damage detection procedure instead of $\Phi$. In the following sections, the case of damage estimation under the assumption of unknown masses will be investigated, evaluating the reliability of the results obtained by using the matrix $\Psi$ instead of $\Phi$ by means of simulated tests.

\section{Damage Detection}

For a precise and reliable estimate of the damage in complex structures, a large number of sensors are generally required $[4,5]$ and the model suitable for damage detection strictly depends on the typology of the analyzed structure. Considering the example of reinforced concrete frames, damage may occur at the columns, beams, or nodes, requiring appropriate assessments for each element. However, usually even in the design phase, it is possible to assume simplified hypotheses at the basis of the model used to perform structural calculations [38]. Moment Resisting Frame (MRF) and other structural typologies can be analyzed, depending on the case, by evaluations made on two limit schemes or a mediated condition.

In particular, if it is possible to model the beams as infinitely stiff, the frame behavior can be assumed as sheartype (Figure 4(a)); that is, it is assumed that the nodes can only translate, by inhibiting rotations. In this case, it is possible to model the structure as a discrete system, where the number of DOFs is equal to the number of allowed translations. The mentioned system can also be modeled as a continuous cantilever beam, deformable only for shear, whose stiffness is obtained by the methods described in the following sections.

If columns are made up of strong elements and the connecting beams can be schematized as weakly bending resistant elements, the structure can be modeled as a set of cantilever beams connected by truss elements (Figure 4(b)).

From the knowledge of natural frequencies and modal mass-normalized matrix, it is possible to obtain the flexibility matrix of the structure $[6,33]$, expressed by the relation:

$$
\mathbf{G}=\overline{\boldsymbol{\Phi}} \boldsymbol{\Omega}^{-2} \overline{\boldsymbol{\Phi}}^{\mathrm{T}}
$$

where $\bar{\Phi} \in \mathbb{R}^{n \times n}$ is the mass-normalized modal matrix and $\boldsymbol{\Omega}=\operatorname{diag}\left\{\omega_{i}\right\}$ is the natural circular frequencies matrix. If the available data is incomplete, or for a number of known vibrational modes of $r<n$, we can estimate the approximate flexibility matrix as

$$
\mathbf{G} \cong \sum_{i=1}^{r} \frac{1}{\omega_{i}^{2}} \bar{\phi}_{i} \bar{\phi}_{i}^{\mathrm{T}},
$$

where $\bar{\phi}_{i}$ is the $i$ th mass-normalized modal vector [39]. By using the vectors $\psi_{i}$, computed by means of the SVD (possibly through a set of repeated tests), we can compute an approximate nonnormalized flexibility matrix as

$$
\boldsymbol{\Gamma}=\sum_{i=1}^{r} \frac{1}{\omega_{i}^{2}} \boldsymbol{\psi}_{i} \boldsymbol{\psi}_{i}^{\mathrm{T}} .
$$

4.1. Shear-Type Frame. Assuming that a unit load vector $\mathbf{p}=$ $[1,1, \ldots, 1]^{\mathrm{T}}$ is applied to the analyzed structure, it is possible to estimate the corresponding displacement vector as

$$
\mathbf{u}=\mathbf{G} \mathbf{p} .
$$


Given the vector (11) it is also possible to compute the interstory drift (ID) $\delta_{j}$ for the $j$ th story, by the difference between two subsequent terms of the $\mathbf{u}$ vector. It is therefore possible to evaluate the variation of the ID between an initial and an eventually damaged condition, in order to determine whether the system has undergone a decrease in stiffness between the two different time instants. In particular, the ID of the $j$ th story caused by the story shear $V_{j}$ can be expressed as

$$
\delta_{j}=V_{j} f_{j}=V_{j} \frac{h_{j}^{3}}{12 E J_{s, j}},
$$

where $f_{j}$ is the $j$ th story flexibility, $h_{j}$ represents the $j$ th story height, $E$ is the elastic modulus of the considered material (supposed as constant for each story), and $J_{s, j}$ is the $j$ th story stiffness (i.e., the sum of column stiffness of the $j$ th story) in the direction of load. In order to obtain the $j$ th story stiffness variation $\varepsilon_{j}$ between the integer and the damaged condition, the relation (12) can be reversed, obtaining

$$
\varepsilon_{j}=\frac{E J_{s, j}-\widetilde{E J_{s, j}}}{E J_{s, j}}=\frac{\widetilde{\delta_{j}}-\delta_{j}}{\widetilde{\delta_{j}}},
$$

where the terms $\tilde{\boldsymbol{q}}$ characterize the damaged structure. Finally, the results of (13) can be grouped into vectors $\boldsymbol{\varepsilon}$ and $\boldsymbol{\delta}$, the elements of which represent, respectively, the variations in story stiffness and the ID of the whole structure. The $j$ th element of $\boldsymbol{\varepsilon}$ can also be expressed as

$$
\varepsilon_{j}=\frac{\left(\widetilde{\mathbf{g}}_{j} \mathbf{p}-\widetilde{\mathbf{g}}_{j+1} \mathbf{p}\right)-\left(\mathbf{g}_{j} \mathbf{p}-\mathbf{g}_{j+1} \mathbf{p}\right)}{\left(\widetilde{\mathbf{g}}_{j} \mathbf{p}-\widetilde{\mathbf{g}}_{j-1} \mathbf{p}\right)},
$$

where $\mathbf{g}_{j}$ is the $j$ th line of the system's flexibility matrix. With reference to relation (9), flexibility matrix can be expressed as a function of the modal vectors normalized to unitary masses:

$$
\mathbf{G} \cong \sum_{i=1}^{r} \frac{\alpha_{i}^{2}}{\omega_{i}^{2}} \phi_{i} \phi_{i}^{\mathrm{T}}
$$

where $\phi_{i}$ is the $i$ th unitary-mass-normalized modal vector and $\alpha_{i}$ is the normalization coefficient [30,31], generally different for each modal vector, obtained as

$$
\alpha_{i}=\left(\phi_{i}^{\mathrm{T}} \mathbf{M} \phi_{i}\right)^{-1 / 2}
$$

where $\mathbf{M}$ is the mass matrix of the structure, supposed as unknown. It is noted that, in traditional flexibility-based damage detection techniques, the normalization coefficients evaluation is necessary. For this reason, if the real distribution of masses is unknown, the approximate assessment of the structural masses involves the introduction of related estimation errors.

Substituting (15) in (14) it is possible to express the elements of the story stiffness variation vector as a function of unitary-mass-normalized modal shapes and natural frequencies of the system, obtained by the identification process conducted at two different time instants. Assuming that modal shapes vary in modest quantities between the integer and the damaged conditions, $\alpha_{i}$ coefficients can be considered as unchanged. Furthermore, if masses are nearly uniformly distributed along the structure, the decrease in stiffness can be evaluated depending on the first $r$ vibration modes identified, through the relation:

$$
\begin{aligned}
& \varepsilon_{j} \\
& \cong \frac{\sum_{i=1}^{r}\left[\left(\left(\widetilde{\phi}_{j, i}-\widetilde{\phi}_{j+1, i}\right) / \widetilde{\omega}_{i}^{2}\right) \widetilde{\boldsymbol{\phi}}_{i}^{\mathrm{T}} \mathbf{p}-\left(\left(\phi_{j, i}-\phi_{j+1, i}\right) / \omega_{i}^{2}\right) \boldsymbol{\phi}_{i}^{\mathrm{T}} \mathbf{p}\right]}{\sum_{i=1}^{r}\left[\left(\left(\widetilde{\phi}_{j, i}-\widetilde{\phi}_{j+1, i}\right) / \widetilde{\omega}_{i}^{2}\right) \widetilde{\boldsymbol{\phi}}_{i}^{\mathrm{T}} \mathbf{p}\right]},
\end{aligned}
$$

where $\mathbf{p}$ represents a unitary vector and $\phi_{j, i}$ the $j$ th element of $\phi_{i}$. Similarly, by using the singular vectors, we can evaluate the decrease in stiffness as

$$
\begin{aligned}
& \epsilon_{j} \\
& \cong \frac{\sum_{i=1}^{r}\left[\left(\left(\widetilde{\psi}_{j, i}-\widetilde{\psi}_{j+1, i}\right) / \widetilde{\omega}_{i}^{2}\right) \widetilde{\psi}_{i}^{\mathrm{T}} \mathbf{p}-\left(\left(\psi_{j, i}-\psi_{j+1, i}\right) / \omega_{i}^{2}\right) \boldsymbol{\psi}_{i}^{\mathrm{T}} \mathbf{p}\right]}{\sum_{i=1}^{r}\left[\left(\left(\widetilde{\psi}_{j, i}-\widetilde{\psi}_{j+1, i}\right) / \widetilde{\omega}_{i}^{2}\right) \widetilde{\psi}_{i}^{\mathrm{T}} \mathbf{p}\right]} .
\end{aligned}
$$

In the Applications section the reliability of relation (18) and the differences between the results obtained by using (18) instead of (17) are investigated.

4.2. Continuous Shear-Deflecting Cantilever Beam. In order to generalize the obtained results, continuous schemes are also studied. If the structure analyzed in the previous case is regular in height, both in terms of mass and stiffness, it can be modeled as a continuous cantilever beam deflecting for shear, where its mass is distributed and whose equivalent stiffness is the mean value over all stories of the terms $(G A)_{e q, j}$ (i.e., the $j$ th segment stiffness), estimated by equating the drift of the two systems shown in Figure 5:

$$
(G A)_{e q, j}=\frac{12 E J_{s, j}}{h_{j}^{2}},
$$

where $h_{j}$ is the $j$ th story height, $E$ is the elastic modulus of the considered material (supposed as constant for each story), and $J_{s, j}$ is the $j$ th story stiffness in the direction of load, as in the previous case.

Applying a number $m$ of sensors at a variable spacing on the analyzed structure, the procedure is similar to the shear-type case, where the order of the system is represented by $m$. Assuming the application of a unitary force vector at the sensors location, the damage index can be estimated as an ID variation, as explained above. It is noted that the localization and quantification of decreasing stiffness depend on the spacing: the greater the number of sensors is, the more precisely the damage position will be located. In addition, the decrease in stiffness, expressed as a percentage of variation, is related to the part of the structure between two sensors: for the same damage, the higher the spacing, the lower the percentage of estimated damage, with the consequent increase in calculation error.

4.3. Continuous Bending Moment-Deflecting Cantilever Beam. In case the structure can be modeled as a continuous cantilever beam deflecting for bending moment (e.g., reinforced 


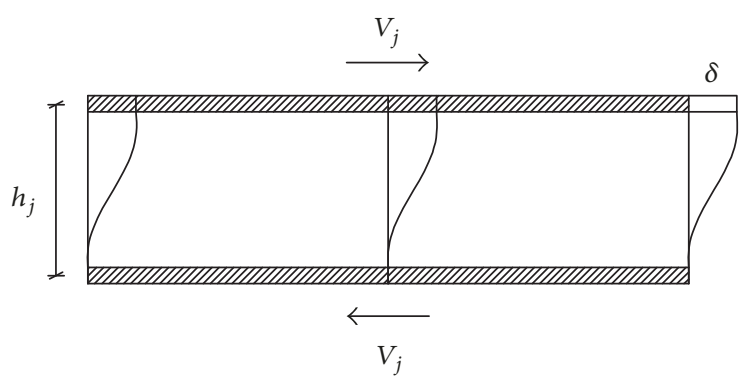

(a)

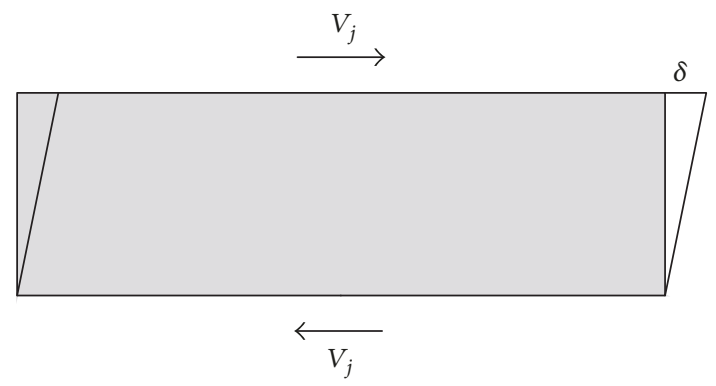

(b)

FIGURE 5: Model used for calculating the translational stiffness of the equivalent shear-deflecting cantilever beam. (a) A shear-type frame story; (b) a continuous element of the same length, subjected to the same shear of the scheme on (a).

concrete walls), each control point can also rotate as a result of load application (Figure 4(b)). By assuming that the sensors are close enough, the structure can be modeled as a set of beam segments with different stiffness values, delimited by control points. In this case, it is possible to obtain the flexibility matrix and the displacement vectors of the control points subjected to unitary loads, similarly to the previous cases. However, since nodes are also allowed to rotate, the $u_{j}$ displacement has to be cleaned of the term associated with the rigid rotation $\theta_{j}$ of the structure above the $j$ th node, caused by the whole set of loads applied on the structure. It is therefore necessary to perform a recursive procedure, initially evaluating the rotation of the first control point from the base, knowing the $u_{1}$ displacement of the same point:

$$
\theta_{1}=\frac{3 u_{1}}{h_{1}}\left(\frac{h_{1} m+2 \sum_{i=1}^{m}\left(h_{i}-h_{1}\right)}{2 h_{1} m+3 \sum_{i=1}^{m}\left(h_{i}-h_{1}\right)}\right)
$$

where $m$ is the number of sensors applied to the structure and $h_{j}$ is the distance of the $j$ th sensor from the base of the structure. Considering for simplicity that the sensors are placed at constant spacing $s$, the relation (20) becomes

$$
\theta_{1}=\frac{6 m u_{1}}{s(1+3 m)} \text {. }
$$

The upper part of the structure will therefore be subject to a rigid rotation of $\theta_{1}$, in addition to the elastic deformation caused by the loads above the first control point. The net ID of the second segment is thus evaluated as

$$
\delta_{2}=u_{2}-u_{1}-\theta_{1} s .
$$

This value must be used to obtain the total rotation of the second node, as

$$
\theta_{2}=\theta_{1}+\frac{6(m-1) \delta_{2}}{s[1+3(m-1)]}
$$

It will thus be possible to evaluate the next ID from which the rotation of the same node can be obtained, until the $m$ th term. The estimate of damage is thus evaluated as an $\boldsymbol{\varepsilon}$ vector whose elements have the form:

$$
\varepsilon_{j}=\frac{\widetilde{\delta_{j}}-\delta_{j}}{\widetilde{\delta_{j}}} .
$$

It is important to emphasize that relations (20)-(23) are valid if the stiffness value is constant along the considered segment. This hypothesis is usually not verified if the damage is concentrated in a small section of the structure (e.g., cracking or concentrated lesions, where the element curvature changes abruptly). In this case, relation (24) would provide accurate results only with respect to the first lesion from the base of the structure, as the upper control points rotations would be incorrectly evaluated. This drawback can be overcome by decreasing the discretization interval, so that segments between sensors are small enough to be considered with a constant stiffness along their whole length.

In the case of reduced availability of sensors, however, it is possible to decrease the discretization interval by performing repeated tests with the same sensors in different positions, maintaining one fixed at a time, in order to normalize modal shapes at the same value (Figure 6). In this way, it is possible to increase the number of control points and thus the rank of the matrix $\Gamma$.

It is also noted that the method described in this paragraph is more sensitive to the errors on natural frequencies than the technique described for shear-type structures. This phenomenon is due to the increased overall number of DOFs and can be fixed by averaging the results obtained through the modal identification processes, carried out on different tests with displaced sensors.

In case of systems that can be modeled as several cantilever structures connected together by flexible beams, it is possible to approximate them as truss elements. Under these assumptions, we evaluate the overall stiffness of a flexural deformable equivalent structure as

$$
(E J)_{e q}=\sum_{i=1}^{q} E J_{i}
$$

where $E J_{i}$ is the stiffness of a single cantilever beam and $q$ is the number of interconnected structures.

The described cases represent simple structures whose behavior can be studied in two dimensions. If the examined structures have a more complex behavior (e.g., asymmetrical structures or with eccentricity of the masses), a higher number of sensors could be needed to record the acceleration in multiple directions, in order to study the 


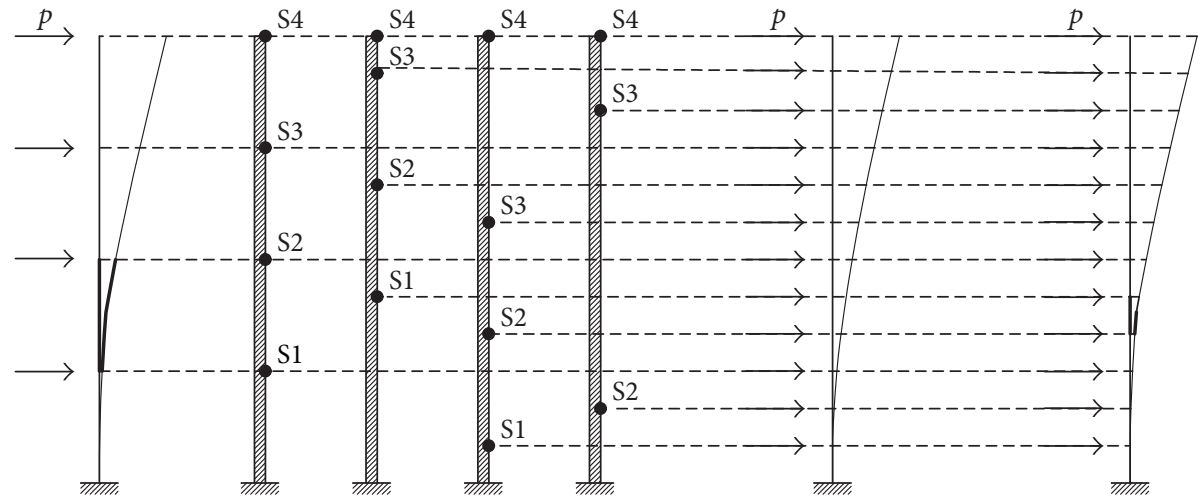

Figure 6: Reduction of the discretization interval through repeated tests.

three-dimensional behavior of the system. If, moreover, the materials have a strongly nonlinear behavior (e.g., historical masonry buildings), the problem becomes more complicated and a precise mechanical characterization must be carried out before choosing the model to be used for damage estimation purposes [40-43].

\section{Applications}

In this section, the effectiveness of the use of singular vectors for damage detection purposes is examined by means of simulated tests on different structural typologies. A comparison between the modal parameters obtained by the traditional ERA and those computed by using the waveletbased procedures is also shown, by using the same dynamic recordings, with the aim of comparing two quantities affected by error. In order to prove the reliability of the methods even for real life cases, in which there is always an error due to the instrumentation sensitivity, for each application a white noise level is artificially introduced into the recordings, as specified for each following case.

In the analyzed examples, the fluctuations of the modal parameters due to environmental mutations, such as changes in temperature or operative conditions, are neglected. However, since the analysis in the time-frequency domain allows the estimation of instantaneous modal parameters over time, under more complex environmental conditions, in order to obtain the clean modal parameters necessary for the estimation of damage, removal techniques could be used, as widely described in literature [40-45]. Therefore, in the following examples we use parameters cleaned up by environmental effects, taking into account the instrumentation sensitivity only.

5.1. Shear-Type Frame. The first case is a lumped-mass reinforced concrete plane frame composed of four stories, each consisting of five equal columns, in which the beams are assumed as infinitely stiff, so that the structure can be modeled as shear-type (Figure 7). The described structure is fully characterized by four vibration modes.

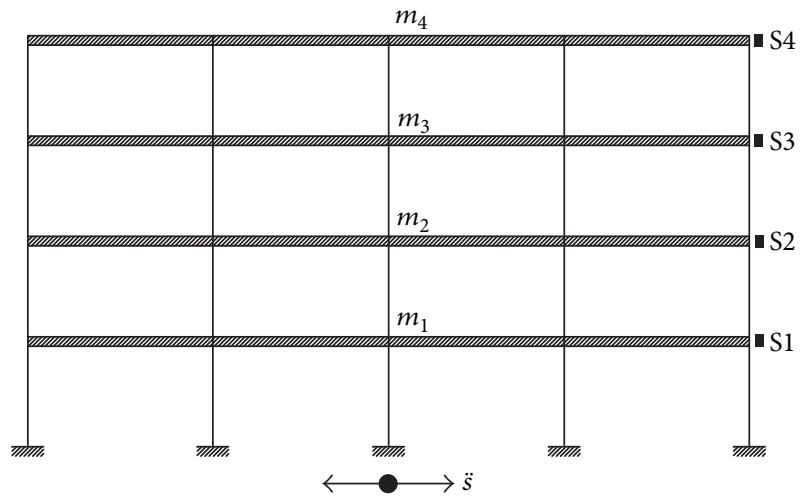

FIGURE 7: Shear-type frame and sensors disposition.

TABLE 1: Geometric data of the analyzed shear-type frame.

\begin{tabular}{lcccc}
\hline Story & $\begin{array}{c}\text { Story mass } \\
{\left[\mathrm{kN} \mathrm{s}^{2} / \mathrm{m}\right]}\end{array}$ & $\begin{array}{c}\text { Story } \\
\text { height } \\
{[\mathrm{m}]}\end{array}$ & $\begin{array}{c}\text { Column } \\
\text { section } \\
{[\mathrm{m}]}\end{array}$ & $\begin{array}{c}\text { Sum of story } \\
\text { columns inertia } \\
{\left[\mathrm{m}^{4}\right]}\end{array}$ \\
\hline 4 & 69.2 & 3.20 & $35 \times 50$ & $1.823 \cdot 10^{-2}$ \\
3 & 95.4 & 3.20 & $40 \times 50$ & $2.083 \cdot 10^{-2}$ \\
2 & 96.4 & 3.20 & $45 \times 50$ & $2.344 \cdot 10^{-2}$ \\
1 & 97.3 & 3.50 & $45 \times 50$ & $2.344 \cdot 10^{-2}$ \\
\hline
\end{tabular}

The mass matrix $\mathbf{M}$ and the stiffness matrix $\mathbf{K}$ are evaluated on the basis of the geometric data (Table 1), taking into account also the other structural and nonstructural components in mass computation. The mass-normalized modal matrix $\bar{\Phi}$ and the natural circular frequency matrix $\boldsymbol{\Omega}$ are obtained by solving the eigenvalue problem for the undamped system in free vibration.

$$
\mathbf{M}=\left[\begin{array}{cccc}
69.2 & 0 & 0 & 0 \\
0 & 95.4 & 0 & 0 \\
0 & 0 & 96.4 & 0 \\
0 & 0 & 0 & 97.3
\end{array}\right] \mathrm{kN} \mathrm{s}^{2} / \mathrm{m}
$$


K

$$
\begin{aligned}
& =\left[\begin{array}{cccc}
133514 & -133514 & 0 & 0 \\
-133514 & 286102 & -152588 & 0 \\
0 & -152588 & 324249 & -171661 \\
0 & 0 & -171661 & 302856
\end{array}\right] \mathrm{kN} / \mathrm{m}, \\
& \overline{\boldsymbol{\Phi}}=\left[\begin{array}{cccc}
-0.0696 & -0.0713 & 0.0616 & -0.0270 \\
-0.0621 & -0.0116 & -0.0600 & 0.0538 \\
-0.0474 & 0.0524 & -0.0234 & -0.0695 \\
-0.0288 & 0.0617 & 0.0592 & 0.0462
\end{array}\right]
\end{aligned}
$$$$
\boldsymbol{\Omega}=\left[\begin{array}{cccc}
14.4431 & 0 & 0 & 0 \\
0 & 40.1943 & 0 & 0 \\
0 & 0 & 61.7279 & 0 \\
0 & 0 & 0 & 75.9607
\end{array}\right] \mathrm{rad} / \mathrm{s} .
$$

Finally, the flexibility matrix $\mathbf{G}$ is obtained as inverse of the stiffness matrix:

$$
\mathbf{G}=\left[\begin{array}{llll}
0.2749 & 0.2000 & 0.1345 & 0.0762 \\
0.2000 & 0.2000 & 0.1345 & 0.0762 \\
0.1345 & 0.1345 & 0.1345 & 0.0762 \\
0.0762 & 0.0762 & 0.0762 & 0.0762
\end{array}\right] \cdot 10^{-4} \mathrm{~m} / \mathrm{kN} \text {. }
$$

5.1.1. Modal Identification. In the first analysis, a stochastic acceleration with nonstationary characteristics is applied at the base of the structure, with reference to the accelerogram shown in Figure 8(b).

By applying the RWS-TSVD-based identification procedure on the time history collected at the sensor S1, on which an error of 5\% (in terms of energy, with respect to the recorded signal) has been introduced, the circular frequencies $\omega_{i}$ are evaluated and reported in Table 2. Furthermore, an SVD of the matrix composed of the whole set of structural dynamic responses has been performed, obtaining the left singular vectors (also shown in Table 2) and the right singular vectors, reported in Figure 9 next to their frequency domain representations. It is observed that the frequency values estimated by the RWS-TSVD-based procedure are approximately coincident with the maximum frequency values of the Fourier spectra computed on the right singular vectors, confirming the fact that the estimated parameters correspond to real vibration modes.

In Figure 10 the features detection procedure is shown in detail: the traditional wavelet scalogram (Figure 10(a)) and the reassigned version (Figure 10(b)) are reported, and since the energy spread is lower in the second case, a more precise features detection (Figure 10(c)) is allowed. It should be noted that the structural masses have not been used during the identification phase.

5.1.2. Error Evaluation and Comparison. A comparison between the identified singular vectors $\psi_{i}$ and analytical

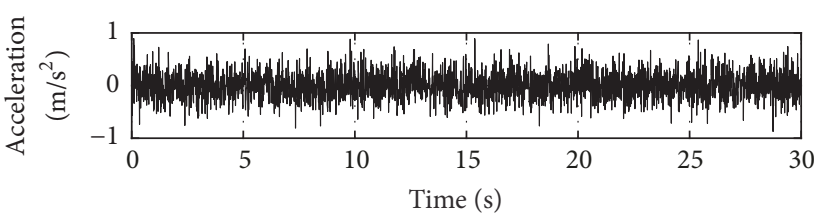

(a)

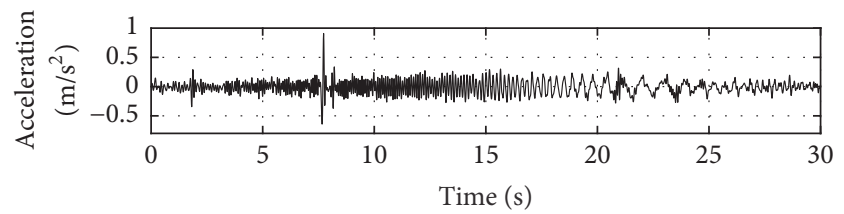

(b)

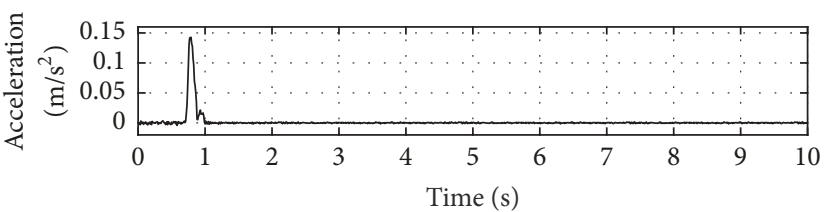

(c)

FIGURE 8: Accelerograms representing a white noise with Gaussian distribution (a), a nonstationary stochastic signal (b), and the modeling of an elastic shock (c).

TABLE 2: Natural frequencies identified by the RWS-TSVD-based algorithm and singular vectors computed for the undamaged frame.

\begin{tabular}{lccccc}
\hline Mode $i$ & $\widehat{\omega}_{i}[\mathrm{rad} / \mathrm{s}]$ & $\widehat{\psi}_{4, i}$ & $\widehat{\psi}_{3, i}$ & $\widehat{\psi}_{2, i}$ & $\widehat{\psi}_{1, i}$ \\
\hline 1 & 14.4463 & 0.6424 & 0.5723 & 0.4360 & 0.2641 \\
2 & 39.9893 & -0.6004 & -0.0774 & 0.5261 & 0.5972 \\
3 & 64.4856 & 0.4519 & -0.6243 & -0.2442 & 0.5886 \\
4 & 77.0482 & -0.2049 & 0.5454 & -0.6722 & 0.4569 \\
\hline
\end{tabular}

TABle 3: Percentage errors on estimated values (normalized to unitary masses). Mean errors are computed on the absolute values of above terms.

\begin{tabular}{lccccc}
\hline Mode $i$ & $\operatorname{Err}\left(\widehat{\omega}_{i}\right)[\%]$ & \multicolumn{4}{c}{$\operatorname{Err}\left(\widehat{\psi}_{j, i}, \phi_{j, i}\right)[\%]$} \\
\hline 1 & +0.22 & +0.15 & +0.01 & -0.18 & -0.48 \\
2 & -0.51 & -9.70 & -28.44 & +7.76 & +3.78 \\
3 & +4.47 & -22.95 & +9.19 & +9.42 & +4.32 \\
4 & +1.43 & -22.27 & +3.96 & -0.90 & +1.46 \\
\hline Mean & 1.66 & 13.77 & 10.40 & 4.57 & 2.51 \\
\hline
\end{tabular}

unitary-mass-normalized modal shapes is reported in Figure 11. With the terms we indicate the quantities estimated by the identification algorithm, thus having an error, as reported in Table 3.

The identification algorithm is general and would allow the correct identification of the examined structure, even in case of flexible beams. In particular, assuming beams of $30 \times$ $50 \mathrm{~cm}$ and removing the hypothesis of infinite stiffness, the average error over all modes in natural frequency evaluation is of $1.87 \%$.

In this section we also compare the error level of the frequencies obtained through the wavelet-based methods 

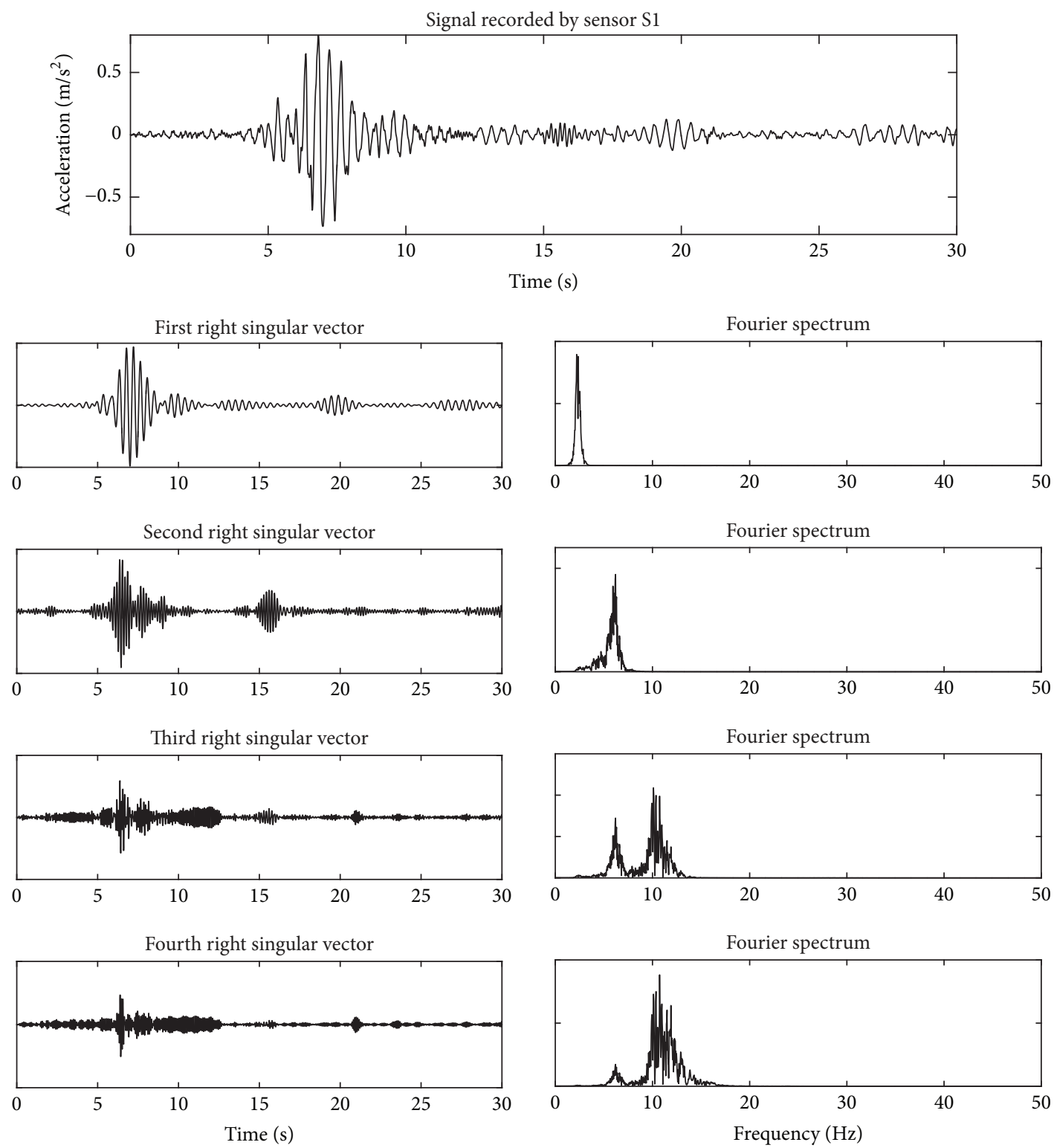

FIgURE 9: First four right singular vectors and their frequency contents.

and the ERA. For this purpose, three different tests are performed, each by using one of the acceleration time histories of Figure 8 as input, for which the natural frequencies of the structure represented in Figure 7 are evaluated. In particular, the time history of Figure 8(a) is a zero-mean white noise with Gaussian distribution, representing stationary ambient vibration applied to the base of the analyzed structure. The accelerogram of Figure $8(\mathrm{~b})$ is a nonstationary stochastic signal which represents a more general ambient excitation, also applied to the base of the structure; meanwhile the input of Figure 8(c) represents an elastic shock applicable to any level of the structure (in this case at the second floor, simulating a forced impulsive vibration test).

The structural responses (collected with a frequency sampling of $100 \mathrm{~Hz}$ ) have then been processed by using the wavelet-based procedures and the ERA, separately. In particular, the results of wavelet-based algorithms are compared to those obtained by means of NExT-ERA, applied to the responses obtained by the input acceleration (a), RD-ERA, applied to the responses obtained by the input acceleration (b), and ERA, applied to the responses obtained by the input acceleration (c) of Figure 8. Lastly, a comparison has been made between the WS-TSVD-based technique, with reference to the traditional scalogram, and the RWS-TSVDbased procedure, with reference to the reassigned one. The first two time histories have a duration of $30 \mathrm{~s}$, while the third has a duration of $10 \mathrm{~s}$.

Observing the results in Table 4, the ERA provides a complete dynamic description for the analyzed system only in the case (c), since the recordings (a) and (b) are too 


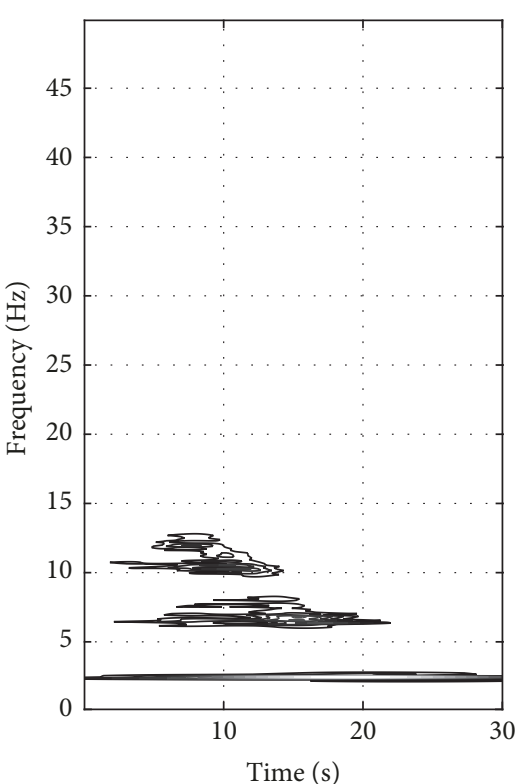

(a)

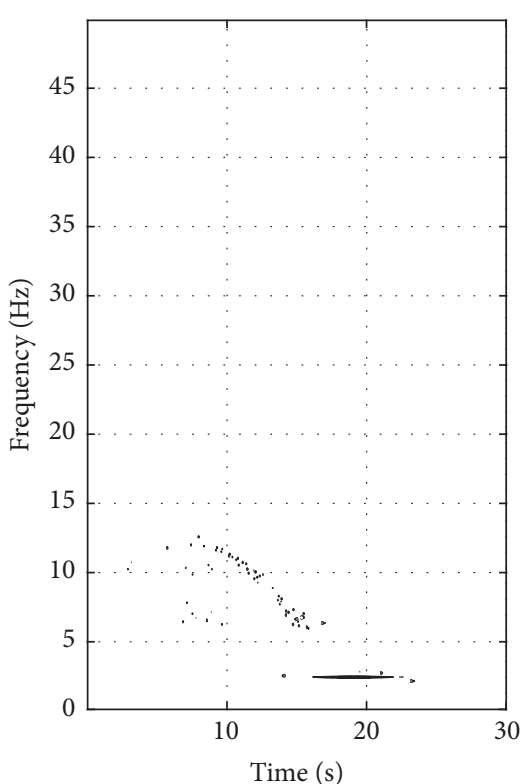

(b)

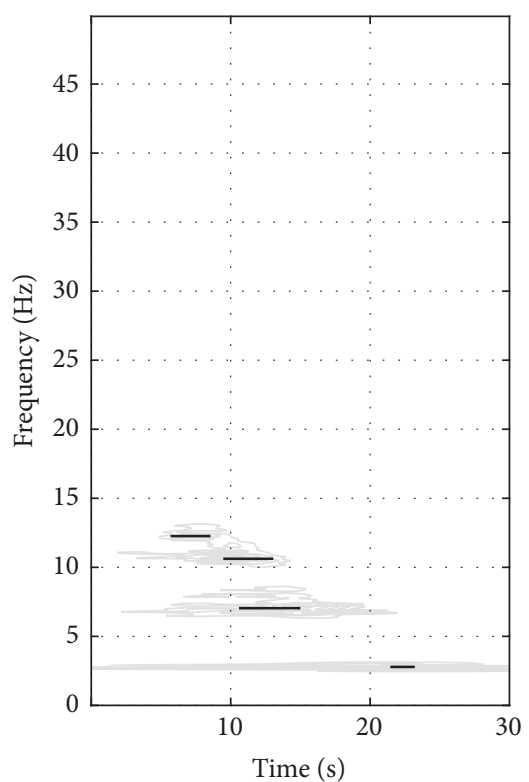

(c)

Figure 10: Morlet scalogram (a), Morlet reassigned scalogram (b), and detected features (c).
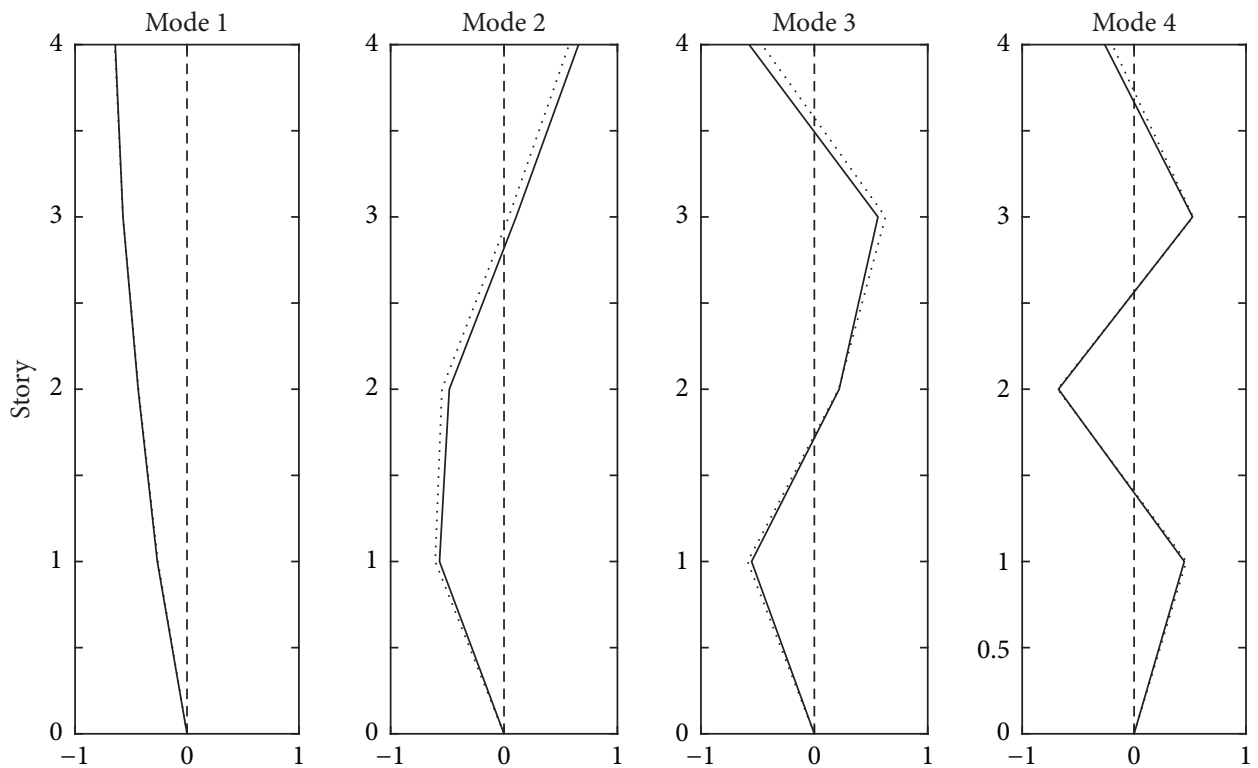

Left singular vectors

Unitary-mass-normalized modal vectors

FIGURE 11: Comparison between identified left singular vectors and analytical modal shapes. In this representation the values are simply connected by continuous or dashed lines (resp., for identified and analytical values) in order to improve the graph readability.

short to allow an efficient execution of RD technique and NExT, while the wavelet-based procedures work properly even with short recordings. It is also noted that in general the reassigned scalogram is more accurate in determining the natural frequencies, especially for the first modes. Moreover, the third column associated with the RWS-TSVD-based method shows an improvement with respect to the WSTSVD-based procedure, as regards the estimation of natural frequencies.

Repeating the tests by using a stationary excitation (as the one of Figure 8(a)), with a duration of 5 minutes, all the modes are identified through the NExT-ERA technique 
TABLE 4: Modal parameters identified by ERA, WS-TSVD-based algorithm, and RWS-TSVD-based algorithm on the undamaged frame, with reference to input accelerations of Figure 8.

\begin{tabular}{|c|c|c|c|c|c|c|c|}
\hline \multirow{2}{*}{ Input } & \multirow{2}{*}{ Mode $i$} & \multicolumn{2}{|c|}{ ERA } & \multicolumn{2}{|c|}{ WS-TSVD } & \multicolumn{2}{|c|}{ RWS-TSVD } \\
\hline & & $\widehat{\omega}_{i}[\mathrm{rad} / \mathrm{s}]$ & Err [\%] & $\widehat{\omega}_{i}[\mathrm{rad} / \mathrm{s}]$ & Err [\%] & $\widehat{\omega}_{i}[\mathrm{rad} / \mathrm{s}]$ & Err [\%] \\
\hline \multirow{4}{*}{ (a) } & 1 & 14.3482 & -0.66 & 14.2788 & -1.14 & 14.2788 & -1.14 \\
\hline & 2 & 41.2713 & +2.68 & 40.8269 & +1.57 & 40.6176 & +1.05 \\
\hline & 3 & - & - & 62.3920 & +1.08 & 62.3920 & +1.08 \\
\hline & 4 & - & - & 78.9325 & +3.91 & 79.1417 & +4.19 \\
\hline \multirow{4}{*}{ (b) } & 1 & 14.6804 & +1.64 & 14.0746 & -2.55 & 14.4463 & +0.02 \\
\hline & 2 & 39.4661 & -1.81 & 41.4552 & +3.14 & 39.9893 & -0.51 \\
\hline & 3 & 66.5500 & +7.81 & 63.6487 & +3.11 & 64.4856 & +4.47 \\
\hline & 4 & - & - & 74.5355 & -1.87 & 77.0482 & +1.43 \\
\hline \multirow{4}{*}{ (c) } & 1 & 14.4551 & +0.08 & 14.4369 & -0.04 & 14.4369 & -0.04 \\
\hline & 2 & 40.3869 & +0.48 & 40.1722 & -0.05 & 40.1722 & -0.05 \\
\hline & 3 & 64.5058 & +4.50 & - & - & 63.3973 & +2.70 \\
\hline & 4 & 72.5101 & -4.54 & - & - & - & - \\
\hline
\end{tabular}

and the obtained results are comparable to those obtained through the RWS-TSVD-based procedure (the average error over all modes is of $1.76 \%$ ), while using a nonstationary input with a duration of 5 minutes, all the modes are identified through the RD-ERA technique, with an average error over all modes equal to $3.74 \%$ and a maximum value of $8.48 \%$. The results obtained through the RWS-TSVD-based procedure, on the other hand, are not dependent on the duration of the recording, since the errors computed on longer tests are similar to those shown in Table 4.

It is observed that in wavelet-based techniques the nature of the excitation does not affect the estimation error on modal frequencies, but the number of estimated modes. In fact, as we can see in Table 4, not all the modes have been identified in the impulsive excitation test. This happens because the frequency spectrum of the input of Figure 8(c) has low values for the range between 7 and $15 \mathrm{~Hz}$ and thus generates structural responses in which the third and fourth vibration modes have low energy level. By performing the wavelet analysis on these responses, the energy peaks associated with the last two modes are low and widespread in the time-frequency plane. For this reason, by using the WSTSVD-based procedure, the peaks associated with the last two modes are classified as noise and therefore discarded by the algorithm. Instead, by applying the RWS-TSVD-based method, the third mode is also identified, since the associated energy peak is sharper in the time-frequency plane and therefore is recognized as representative of a structural mode. The fourth mode would only be identified by lowering the threshold of the RMS-based filter, but in this case, other noise-related modes would also be recognized as structural modes.

In order to obtain good results in terms of frequencies by using the described wavelet-based techniques, an excitation characterized by a sufficiently wide spectrum of frequencies (i.e., that includes the natural frequencies of the analyzed structure) is therefore necessary, regardless of whether it has stationary or nonstationary characteristics over time. As for
TABLE 5: Flexibility matrix obtained by using unitary-massnormalized identified modal vectors (from ERA-based procedure) and relative errors.

\begin{tabular}{lcccc}
\hline & \multicolumn{4}{c}{ Elements of the flexibility matrix } \\
\hline & 0.2329 & 0.1696 & 0.1145 & 0.0650 \\
$\widehat{\mathbf{G}}\left[\mathrm{m} / \mathrm{kN} \cdot 10^{2}\right]$ & 0.1696 & 0.1703 & 0.1136 & 0.0646 \\
& 0.1145 & 0.1136 & 0.1155 & 0.0644 \\
& 0.0650 & 0.0646 & 0.0644 & 0.0663 \\
\hline & 0.00 & 0.08 & 0.54 & 0.66 \\
$\operatorname{Err}(\widehat{\mathbf{G}} / \max \widehat{\mathbf{G}}, \mathbf{G} / \max \mathbf{G})[\%]$ & 0.08 & 0.50 & -0.25 & 0.02 \\
& 0.54 & -0.25 & 1.39 & -0.25 \\
& 0.66 & 0.02 & -0.25 & 2.61 \\
\hline
\end{tabular}

the singular vectors instead, the best results are obtained for impulsive excitation, that is, in the case of free vibration.

In order to set up the damage detection procedure, the flexibility matrix $\mathbf{G}$ or $\boldsymbol{\Gamma}$ has to be computed. Assuming that the mass matrix is unknown, one of the simplest criteria to mass-normalize the modal matrix is that of assuming a constant distribution of masses along the structure. In order to reduce the error due to the nature of input time histories, ten repeated tests are carried out. For each test, unitarymass-normalized vectors and left singular vectors are used to compute, respectively, $\widetilde{\mathbf{G}}$ and $\widetilde{\boldsymbol{\Gamma}}$, whose mean values (of each element, computed over the whole set of experiments) and errors are reported in Tables 5 and 6. Errors are evaluated between estimated matrices $\widetilde{\mathbf{G}}, \widetilde{\Gamma}$ and the analytic matrix $\mathbf{G}$-reported at (27) - dividing each for its maximum value, in order to assess the ratios between the elements of each matrix.

It is observed from Tables 5 and 6 that the errors evaluated for the $\widehat{\Gamma}$ matrix are on average greater than those estimated on the $\widehat{\mathbf{G}}$ matrix. This happens because the masses of the analyzed structure are rather uniformly distributed and the unitary normalization of modal vectors produces an accurate 
TABLE 6: Flexibility matrix obtained by using identified left singular vectors (from SVD-based procedure) and relative errors.

\begin{tabular}{lcccc}
\hline & \multicolumn{4}{c}{ Elements of the flexibility matrix } \\
\hline & 0.2273 & 0.1688 & 0.1144 & 0.0643 \\
$\widehat{\Gamma}\left[\mathrm{m} / \mathrm{kN} \cdot 10^{2}\right]$ & 0.1688 & 0.1726 & 0.1145 & 0.0642 \\
& 0.1144 & 0.1145 & 0.1177 & 0.0660 \\
& 0.0643 & 0.0642 & 0.0660 & 0.0674 \\
\hline & 0.00 & 2.09 & 2.94 & 2.11 \\
$\operatorname{Err}(\widehat{\mathbf{\Gamma}} / \max \widehat{\boldsymbol{\Gamma}}, \mathbf{G} / \max \mathbf{G})[\%]$ & 2.09 & 4.37 & 3.00 & 1.93 \\
& 2.94 & 3.00 & 5.90 & 4.70 \\
& 2.11 & 1.93 & 4.70 & 6.92 \\
\hline
\end{tabular}

estimate of the modal matrix. These errors may have a limited influence on the results of damage detection: this aspect is the object of the following section.

5.1.3. Damage Detection. In order to assess the reliability of using singular vectors instead of modal vectors for the purposes of damage detection under the assumption of unknown masses, four different examples of damaged structure are analyzed. Consider the structure represented in Figure 7, with damaged columns, according to the patterns described in Table 7. For the sake of brevity, the modal parameters estimated in a single identification procedure (by using the RWS-TSVD-based approach for the estimation of natural frequencies and SVD for the extraction of singular vectors) are reported in Table 8 only for the damage pattern D1. It should also be noted that the tests for the damaged condition are carried out by using inputs uncorrelated to the ones used for the undamaged condition. We indicate with $\tilde{\mathbf{q}}$ the terms related to the damaged structure, obtained by identification tests.

From the identified parameters, it is possible to evaluate the matrices $\widetilde{\mathbf{G}}$ and $\widetilde{\boldsymbol{\Gamma}}$ (assuming a homogeneous distribution of masses along the structure) associated with the damaged frame and, by using relations (17)-(18), the percentage losses in the story stiffness. Ten repeated tests are performed, each by using a 30-second segment of a 5-minute time history, in order to avoid errors due to the nature of each input segment. In Table 9, the mean values of stiffness decrease, computed for each test by using the RWS-TSVD-based algorithm and the singular vectors, are reported, in the case of complete and partial identification, for which it is assumed that only the first vibration mode is detected, while in Table 10 the results obtained by using ERA are reported, as an example of modal vectors-based procedures.

By selecting the negative terms only, as the positive ones indicate an increase in stiffness and are due to estimation errors, the SVD-based method is particularly effective in both localization and damage quantification, with a maximum evaluation error of $0.79 \%$, even in case of partial identification. The errors obtained by the two approaches for the damage detection are absolutely comparable, despite the different errors on flexibility matrices computed in the previous section (Tables 5 and 6).

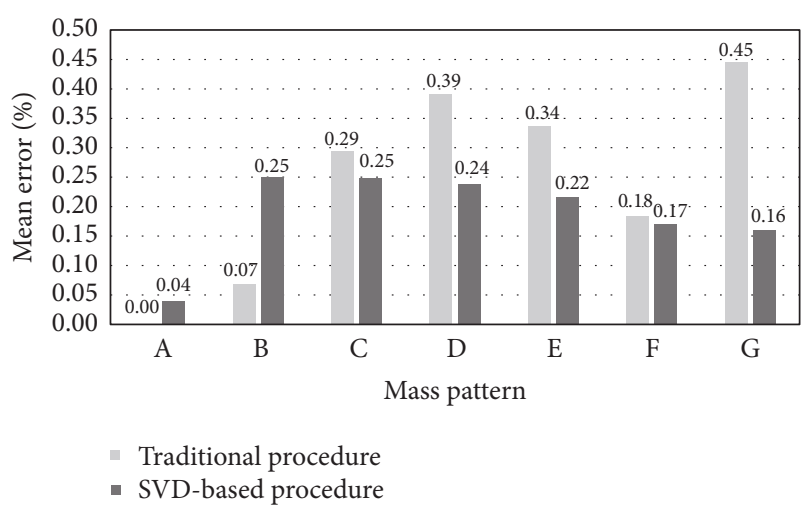

FIGURE 12: Comparison of average absolute errors on analytical damage indices for the shear-type frame, computed by traditional modal vectors-based procedure and SVD-based procedure with reference to the mass patterns of Table 11 and damage pattern D3 of Table 7.

In order to prove, independently of the instrumentation sensitivity, that the SVD-based method works efficiently for the damage detection if masses are supposed as equally distributed when the real distribution is different, Figure 12 shows the mean errors (over all DOFs, for each mass pattern described in Table 11) of damage indices obtained by using analytical parameters instead of the identified ones. The tests are performed on the structure analyzed in the previous example, with different mass patterns and a multiple damage condition represented by the pattern D3 of Table 7 .

Regarding the modal vectors-based procedure, the flexibility matrices have been calculated by using the analytic matrices $\boldsymbol{\Omega}$ and $\boldsymbol{\Phi}$, normalized to unitary masses, while the results concerning the SVD-based procedure are computed by using the analytic matrix $\Omega$ and the left singular vectors, obtained from the impulse response functions of the considered shear-type frame, without adding noise to the recordings (Figure 3).

In Figure 13 the average percentage errors on the flexibility matrix for the damaged condition, computed through the two analyzed approaches, are reported. The error is evaluated as the average of the errors calculated on each element of the matrix. It is noted that these values are independent from those computed for the damage indices.

The mean error on damage indices (over all the tests, with reference to Table 11) for the classical procedure is of $0.25 \%$, while for the SVD-based procedure it is of $0.19 \%$. Since these values are very close and low, it emerges that both relations (17) and (18) work properly for the purposes of damage detection, independently of the error of flexibility matrices. Furthermore, the SVD-based approach does not require any introduction of additional estimated quantities. Indeed, while $\phi_{i}$ vectors have to be mass-normalized, and in the absence of the knowledge of structural masses, additional error could be introduced by a wrong assumption on the mass matrix, and $\psi_{i}$ vectors are already mass-representative. In fact, it can be seen that SVD-based procedure suffers from slightly lower errors than the classical one, in most cases where the masses are not uniformly distributed. It is also noted that $\psi_{i}$ vectors are 
TABLE 7: Damage patterns. The description is referred to as a single column per story.

\begin{tabular}{lcccc}
\hline Damage pattern & Description of the damage in the columns & $\varepsilon_{1}$ & $\varepsilon_{2}[\%]$ & $\varepsilon_{3}$ \\
\hline D1 & $15 \%$ at the 1st story & 3 & 0 & 0 \\
D2 & $15 \%$ at the 2nd story & 0 & 3 & 0 \\
D3 & $15 \%$ at the 1st and 10\% at the 2nd story & 3 & 2 & 0 \\
D4 & $15 \%$ at the 1st, 15\% at the 2nd, and 30\% at the 3rd story & 3 & 3 & 6 \\
\hline
\end{tabular}

TABLE 8: Modal parameters identified for the damaged frame, according to the pattern D1 of Table 7. Natural frequencies are identified by the RWS-TSVD-based algorithm.

\begin{tabular}{lccccc}
\hline Mode $i$ & $\widetilde{\omega}_{i}[\mathrm{rad} / \mathrm{s}]$ & $\widetilde{\psi}_{4, i}$ & $\widetilde{\psi}_{3, i}$ & $\widetilde{\psi}_{2, i}$ & $\widetilde{\psi}_{1, i}$ \\
\hline 1 & 14.4441 & 0.6474 & 0.5715 & 0.4328 & 0.2586 \\
2 & 39.7741 & 0.5831 & 0.0267 & -0.5515 & -0.5959 \\
3 & 60.3263 & 0.4683 & -0.6795 & -0.1313 & 0.5493 \\
4 & 77.1488 & 0.1468 & -0.4592 & 0.7009 & -0.5257 \\
\hline
\end{tabular}

TABLE 9: Damage indices estimated by SVD-based procedure in case of complete (on the left) and partial identification (on the right, by using only the first vector) on the shear-type frame.

\begin{tabular}{cccccc}
\hline & Damage pattern & \multicolumn{4}{c}{ Story } \\
& & 1 & 2 & 3 & 4 \\
\hline \multirow{4}{*}{$\widehat{\epsilon}_{j}[\%]$} & D1 & -2.59 & +0.05 & -0.09 & -0.26 \\
& D2 & -0.50 & -3.61 & -0.39 & -0.25 \\
& D3 & -3.38 & -2.25 & -0.46 & -0.21 \\
& D4 & -3.71 & -3.54 & -6.51 & -0.42 \\
\multirow{4}{*}{$\widehat{\epsilon}_{j}^{(p)}[\%]$} & D1 & -2.23 & +0.73 & +0.99 & +1.01 \\
& D2 & -0.65 & -3.62 & -0.03 & -0.01 \\
& D3 & -2.73 & -1.57 & +0.93 & +0.85 \\
& D4 & -2.33 & -2.21 & -5.65 & +1.23 \\
\hline
\end{tabular}

TABLE 10: Damage indices estimated by modal vectors-based procedure in case of complete (on the left) and partial identification (on the right, by using only the first vector) on the shear-type frame.

\begin{tabular}{cccccc}
\hline & \multirow{2}{*}{ Damage pattern } & \multicolumn{4}{c}{ Story } \\
& & 1 & 2 & 3 & 4 \\
\hline \multirow{4}{*}{$\widehat{\varepsilon}_{j}[\%]$} & D1 & -3.95 & +0.08 & +0.37 & -0.30 \\
& D2 & -1.09 & -3.57 & -0.36 & -0.29 \\
& D3 & -3.42 & -2.65 & +0.18 & -1.02 \\
& D4 & -3.84 & -3.92 & -7.51 & -0.38 \\
\hline \multirow{3}{*}{$\widehat{\varepsilon}_{j}^{(p)}[\%]$} & D1 & -1.62 & +0.42 & +1.04 & +1.02 \\
& D2 & -1.03 & -2.69 & -0.36 & -0.12 \\
& D3 & -2.64 & -1.51 & +0.94 & +0.95 \\
& D4 & -2.22 & -2.29 & -5.48 & +1.35 \\
\hline
\end{tabular}

considerably simpler to be computed than $\phi_{i}$ vectors, which require the use of complex algorithms.

5.2. Continuous Structures. With the aim of proving the generality of the outlined methods, the identification procedure and the damage detection algorithm are also applied
TABLE 11: Mass patterns used for the comparison of the damage errors reported in Figure 12

\begin{tabular}{cccccc}
\hline & Mass pattern & \multicolumn{4}{c}{ Story } \\
& & 1 & 2 & 3 & 4 \\
\hline & A & 100 & 100 & 100 & 100 \\
& B & 50 & 100 & 50 & 100 \\
Mass pattern $\left[\mathrm{kN} \mathrm{s}^{2} / \mathrm{m}\right]$ & $\mathrm{C}$ & 50 & 50 & 100 & 100 \\
& D & 50 & 50 & 100 & 150 \\
& E & 50 & 150 & 150 & 100 \\
& F & 100 & 50 & 100 & 150 \\
& G & 50 & 50 & 50 & 150 \\
\hline
\end{tabular}

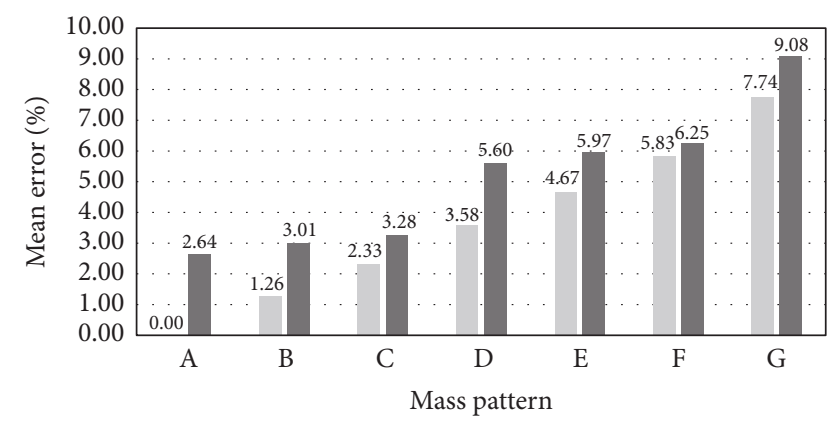

- Traditional procedure
- SVD-based procedure

FIGURE 13: Comparison of average absolute errors on analytical flexibility matrix of the damaged condition, computed by traditional modal vectors-based procedure and SVD-based procedure with reference to the mass patterns of Table 11 and damage pattern D3 of Table 7.

to continuous structures. The accelerogram of Figure $8(\mathrm{~b})$ is used for modal identification purposes while, for the damage detection, a set of ten repeated tests with input stochastic acceleration, not related to the time history (b), are used.

5.2.1. Shear-Deflecting Cantilever Beam. The first analyzed continuous structure consists in a shear-deflecting cantilever beam with homogeneously distributed mass (all the geometric parameters are reported in Table 12). Modal identification is performed on the time history collected by the sensor S2 (positioned as in Figure 14, where $s$ is a constant spacing between the sensors) and by exciting the structure with the accelerogram of Figure 8(b) as a ground acceleration. Other time histories are collected from sensors S1 to S8, in order to carry out damage detection tests. Each recording is affected 


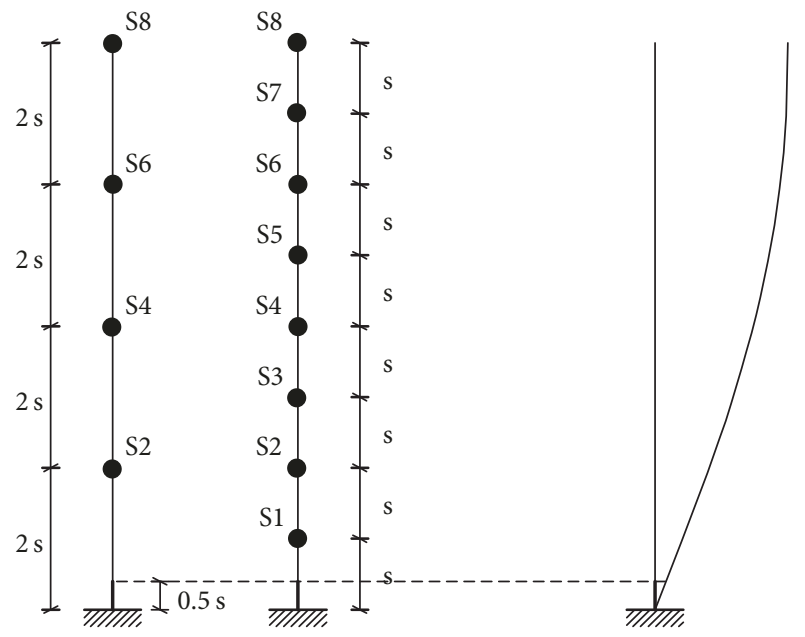

FIgURE 14: Sensors arrangement and first modal shape of the continuous structure.

TABLE 12: Geometric parameters of the undamaged shear-deflecting cantilever beam.

\begin{tabular}{lcc}
\hline$m\left[\mathrm{kN} \mathrm{s}^{2} / \mathrm{m}^{2}\right]$ & $G A[\mathrm{kN}]$ & $\begin{array}{c}H[\mathrm{~m}] \\
\text { Linearly distributed mass }\end{array}$ \\
\hline 26 & Shear stiffness & Total height \\
\hline
\end{tabular}

TABLE 13: Modal parameters estimated on the undamaged continuous shear-deflecting structure by using the RWS-TSVD-based algorithm.

\begin{tabular}{lccc}
\hline Mode $i$ & $\omega_{i}[\mathrm{rad} / \mathrm{s}]$ & $\widehat{\omega}_{i}[\mathrm{rad} / \mathrm{s}]$ & $\operatorname{Err}[\%]$ \\
\hline 1 & 11.4806 & 11.4944 & +0.12 \\
2 & 34.4419 & 35.3581 & +2.66 \\
3 & 57.4033 & 59.2345 & +3.19 \\
4 & 80.3646 & 83.3783 & +3.75 \\
\hline
\end{tabular}

TABle 14: Damage evaluation in case of 4 and 8 sensors on the continuous shear-deflecting structure, by using SVD-based algorithm.

\begin{tabular}{lcccccccc}
\hline Sensor & $\widehat{\epsilon}_{1}$ & $\widehat{\epsilon}_{2}$ & $\widehat{\epsilon}_{3}$ & $\widehat{\epsilon}_{4}$ & $\widehat{\epsilon}_{5}$ & $\widehat{\epsilon}_{6}$ & $\widehat{\epsilon}_{7}$ & $\widehat{\epsilon}_{8}$ \\
\hline Case 1 & - & -7.43 & - & -1.26 & - & +0.86 & - & -1.85 \\
Case 2 & -14.81 & -0.71 & +1.16 & +3.54 & +2.29 & -2.09 & +1.55 & +1.06 \\
\hline
\end{tabular}

by an artificial added noise of $5 \%$ with respect to the collected signals (in terms of energy).

The modal identification results for the undamaged structure are reported in Table 13, with the comparison to analytical values and the relative errors. Two different tests are conducted for the damage detection, by considering 4 and 8 equally spaced sensors along the structure (Figure 14).

Assuming damage at the base of the structure, which can be modeled as a stiffness decrease of the first segment $0.5 \mathrm{~s}$ long (Figure 14), equal to $20 \%$ of its total stiffness, the damage detection procedure should evaluate a $11 \%$ decrease for the 8sensor analysis and a $6 \%$ decrease for the 4 -sensor analysis. The results of these tests are reported in Table 14.
TABLE 15: Geometric parameters of the undamaged bending moment-deflecting cantilever beam.

\begin{tabular}{lcc}
\hline$m\left[\mathrm{kN} \mathrm{s}^{2} / \mathrm{m}^{2}\right]$ & $E J\left[\mathrm{kN} \mathrm{m}^{2}\right]$ & $\begin{array}{c}H[\mathrm{~m}] \\
\text { Linearly distributed mass }\end{array}$ \\
\hline 26 & 200000 & Total height \\
\hline
\end{tabular}

TABLE 16: Modal parameters estimated on the undamaged continuous bending moment-deflecting structure by using the RWS-TSVDbased algorithm.

\begin{tabular}{lccc}
\hline Mode $i$ & $\omega_{i}[\mathrm{rad} / \mathrm{s}]$ & $\widehat{\omega}_{i}[\mathrm{rad} / \mathrm{s}]$ & $\operatorname{Err}[\%]$ \\
\hline 1 & 2.0197 & 1.9672 & -2.60 \\
2 & 13.5253 & 13.5064 & -0.14 \\
3 & 37.5704 & 38.0062 & +1.16 \\
4 & 73.6379 & 70.4494 & -4.33 \\
\hline
\end{tabular}

TABLE 17: Damage evaluation on the continuous bending momentdeflecting structure, by using SVD-based algorithm.

\begin{tabular}{lcc}
\hline Segment $i$ & $\widehat{\epsilon}_{j}[\%]$ & $\operatorname{Err}[\%]$ \\
\hline 1 & -1.34 & -1.34 \\
2 & +5.56 & +5.56 \\
3 & +4.18 & +4.18 \\
4 & -17.88 & -2.12 \\
\hline
\end{tabular}

It should be noted that the second identification case can be obtained by using a smaller number of sensors and performing repeated tests, each by displacing all the accelerometers except one, necessary for normalizing modal vector elements to the same value. In this way, it is possible to increase the discretization level of the analyzed structures in order to better assess the localization of the damage. The errors reported in Table 14 are higher with respect to the errors estimated in the previous simple case (the maximum error is of $3.81 \%$ ). However, the procedure still works for damage detection purposes and further tests may be conducted if necessary.

\subsubsection{Continuous Bending Moment-Deflecting Cantilever} Beam. Modal identification and damage detection procedures are finally performed on a bending moment-deflecting cantilever beam (whose parameters are reported in Table 15), on which 4 equally spaced accelerometers are arranged, starting at $+3.00 \mathrm{~m}$ from the base (as in Figure 14, on the left). As in the previous paragraphs, collected signals are affected by a $5 \%$ noise (in terms of energy, with respect to the registered signals). The evaluated modal parameters are reported in Table 16 together with the respective estimation errors.

Assuming damage at the base of the structure (affecting the segment between 0.0 and $+3.0 \mathrm{~m}$ ), which can be modeled as a $20 \%$ decrease in stiffness, the analysis results are reported in Table 17.

In a further analysis, it is assumed that there are two damaged segments, the first of which is between $+2.0 \mathrm{~m}$ and $+3.0 \mathrm{~m}$, with a $30 \%$ reduction in overall stiffness, and the 
TABLE 18: Damage estimation in case of 4 repeated tests using 4 sensors, I iteration.

\begin{tabular}{|c|c|c|c|c|c|c|c|c|c|c|c|c|}
\hline Height [m] & +1 & +2 & +3 & +4 & +5 & +6 & +7 & +8 & +9 & +10 & +11 & +12 \\
\hline$\varepsilon_{j}[\%]$ & 0 & 0 & -30 & 0 & 0 & -15 & 0 & 0 & 0 & 0 & 0 & 0 \\
\hline$\widehat{\epsilon}_{j}[\%]$ & +2.9 & -3.1 & -28.3 & +2.3 & +2.8 & -14.5 & -33.8 & - & - & - & - & - \\
\hline
\end{tabular}

TABLE 19: II iteration local damage estimation.

\begin{tabular}{lcccccc}
\hline Height $[\mathrm{m}]$ & +5.0 & +5.5 & +6.0 & +6.5 & +7.0 & \\
\hline$\varepsilon_{j}[\%]$ & 0 & 0 & -50 & 0 & 0 & +7.5 \\
$\widehat{\epsilon}_{j}[\%]$ & +1.9 & -1.4 & -48.9 & +4.6 & -1.4 & -3.5 \\
\hline
\end{tabular}

second one between the $+5.5 \mathrm{~m}$ and $+6.0 \mathrm{~m}$, with a $50 \%$ reduction in overall stiffness. Performing the analysis with the sensor arrangement used in the previous case, only the first damage from the base would be properly evaluated, as the rotations computed for the upper control points would be inaccurate. By performing a set of four repeated tests on the damaged structure, each after displacing the sensors (Figure 6), a flexibility matrix of order 12 is obtained. The identified damage indices compared to the theoretical ones are reported in Table 18. It is noted that up to the height of $+5.0 \mathrm{~m}$ the identification is correct, while beyond the $+6.0 \mathrm{~m}$ height invalid values are recorded (i.e., positive, across the allowed error range).

By carrying out further tests and installing the sensors $\mathrm{S} 3$, $\mathrm{S} 2$, and $\mathrm{S} 1$ at $+5.5 \mathrm{~m},+6.5 \mathrm{~m}$, and $+7.5 \mathrm{~m}$, a local analysis can be performed to identify the damage with a sensitivity range of $0.5 \mathrm{~m}$ (Table 19).

As expected, the errors evaluated in the tests carried out in this paragraph are on average higher than those of the previous cases. However, the more the discretization interval is reduced (by performing a large set of repeated tests), the more the obtained results are accurate. In fact, after a preliminary test to locate the damaged areas, it is possible to perform subsequent local tests by providing all available sensors in the concerned segment.

\section{Conclusions}

Two aspects have been addressed in this paper: the first, which is the main purpose, is to study the effectiveness of using singular vectors within a flexibility-based damage detection procedure if the structural masses are unknown; the second is to evaluate the improvements in modal parameters estimation by using the energy reassignment into the framework of a wavelet-based identification procedure. These topics, in particular, have been addressed under nonstationary excitation, for the case of reduced availability of sensors, for which the wavelet-based procedure is also useful for signal sparsification.

As concerns the use of singular vectors as damage sensitive parameters, it has been shown that the results of SVD-based damage estimation are comparable with those obtained by modal matrices. In some cases, the SVD-based damage detection has provided also better results, especially for nonuniformly distributed masses, even if the flexibility matrices computed on singular vectors are on average less precise than those evaluated by modal matrices. It is further observed that singular vectors are generally obtainable in a simpler way with respect to modal vectors. Moreover, by means of the described wavelet-based sparsification technique, they can also be calculated in the case of a limited number of sensors.

Since the sparsification procedure allows a correct estimation for natural frequencies (also necessary to build the flexibility matrix) even under nonstationary excitation, the aspect of dynamic identification has been deepened. In this context a variation to a wavelet-based algorithm described in the literature has been proposed by introducing the energy reassignment. A comparison has been made between the results obtained by means of the RWS-TSVD-based procedure, the original WS-TSVD-based algorithm, and other classical identification techniques capable of computing natural frequencies and modal vectors (for which the ERA has been chosen since it is still one of the most used algorithms in the identification field).

These comparisons have shown that wavelet-based techniques are reliable even in the case of short and nonstationary recordings. However, in order to eliminate the errors in natural frequencies due to the type of excitation and the sensitivity of sensors, several repeated tests have been performed, with the aim of obtaining averaged parameters that are as sensitive as possible only to the changes in structural characteristics. Moreover, in the case of reduced availability of instrumentation, such repeated tests can be performed by displacing the sensors, so as to obtain a more precise discretization and a higher order of the flexibility matrix.

The described procedures are applicable to a wide range of structural schemes, some of which have been studied in the applications part: several tests have been conducted in case of single and multiple damage, obtaining reliable results on both discrete and continuous structures.

\section{Conflicts of Interest}

The authors declare that they have no conflicts of interest.

\section{References}

[1] A. Rytter, Vibrational Based Inspection of Civil Engineering Structures [Ph.D. thesis], Department of Building Technology and Structural Engineering, Aalborg University, Fracture and Dynamics, Aalborg, 1993, vol. R9314, no. 44. 
[2] T. S. Soderstorm and P. G. Stoica, System Identification, Prentice Hall International, 1989.

[3] L. Ljung, System Identification-Theory for the User, Prentice Hall, NJ, USA, 1999.

[4] J. J. Sinou, A Review of Damage Detection and Health Monitoring of Mechanical Systems from Changes in the Measurement of Linear and Non-Linear Vibrations, Mechanical Vibrations: Measurement, Effects and Control, Nova Science Publishers Inc, 2009.

[5] E. Parloo, P. Guillaume, and M. Van Overmeire, "Damage assessment using mode shape sensitivities," Mechanical Systems and Signal Processing, vol. 17, no. 3, pp. 499-518, 2003.

[6] A. K. Chopra, Dynamics of Structures, Prentice Hall, NJ, USA, 2000.

[7] D. J. Ewins, Modal Testing: Theory, Practice and Application, John Wiley \& co, 1998.

[8] D. F. Giraldo, W. Song, S. J. Dyke, and J. M. Caicedo, "Modal identification through ambient vibration: comparative study," Journal of Engineering Mechanics, vol. 135, no. 8, pp. 759-770, 2009.

[9] A. Sadhu, S. Narasimhan, and J. Antoni, "A review of outputonly structural mode identification literature employing blind source separation methods," Mechanical Systems and Signal Processing, vol. 94, pp. 415-431, 2017.

[10] M. R. Hamtaei and S. A. Anvar, Estimation of Modal Parameters of Buildings by Wavelet Transform, Shiraz University, Shiraz, 2008.

[11] Y. Guo and A. Kareem, "System identification through nonstationary response: wavelet and transformed singular value decomposition-based approach," Journal of Engineering Mechanics, vol. 141, no. 7, Article ID 04015004, p. 04015013, 2015.

[12] D. E. Newland, An Introduction to Random Vibrations, Spectral Wavelet Analysis, Dover Publications, New York, NY, USA, 3rd edition, 1993.

[13] L. Cohen, "The wavelet transform and time-frequency analysis," in Wavelets and Signal Processing, Appl. Numer. Harmon. Anal., pp. 3-22, Birkhäuser, Boston, MA, USA, 2003.

[14] A. Graps, "An introduction to wavelets," IEEE Computational Science \& Engineering, vol. 2, no. 2, pp. 50-61, 1995.

[15] T. Kijewski and A. Kareem, "Wavelet transforms for system identification in civil engineering," Computer-Aided Civil and Infrastructure Engineering, vol. 18, no. 5, pp. 339-355, 2003.

[16] J. Lardies and S. Gouttebroze, "Identification of modal parameters using the wavelet transform," International Journal of Mechanical Sciences, vol. 44, no. 11, pp. 2263-2283, 2002.

[17] A. T. Poyil, S. Aljahdali, and K. M. Nasimudeen, "Significance of Cohen's class for time frequency analysis of signals," International Journal of Computer Applications, vol. 72, no. 12, pp. 1-8, 2013.

[18] F. Auger, P. Flandrin, P. Gonçalvès, and O. Lemoine, TimeFrequency Toolbox - for Use with MATLAB, CNRS (France) and Rice University, USA, 1996.

[19] G. D. Clifford, "Singular value decomposition independent component analysis for blind source separation," Journal of Engineering Mechanics, vol. 141, no. 7, 2005.

[20] M. J. Brenner, "Non-stationary dynamics data analysis with wavelet-SVD filtering," Mechanical Systems and Signal Processing, vol. 17, no. 4, pp. 765-786, 2003.

[21] D. Groutage and D. Bennink, "Feature sets for nonstationary signals derived from moments of the singular value decomposition of cohen-posch (positive time-frequency) distributions,"
IEEE Transactions on Signal Processing, vol. 48, no. 5, pp. 14981503,2000

[22] R. Bashor and A. Kareem, "Efficacy of time-frequency domain system identification scheme using transformed singular value decomposition," in Proceedings of the 12th International Conference on Wind Engineering, AWES, pp. 543-550, Cairns, Australia, 2007.

[23] A. C. Bentz, Dynamics of Tall Buildings: Full-Scale Quantification and Impacts on Occupant Comfort [Ph.D. thesis], University of Notre Dame, Notre Dame, IN, USA, 2012, Ph.D. dissertation.

[24] D. Groutage and D. Bennink, "New matrix decomposition based on transforming the basis sets of the singular value decomposition yields principal features for time-frequency distributions," in SPIE Proceedings of Advanced Signal Processing Algorithms, Architectures and Implementations X, vol. 4116, pp. 66-79, San Diego, Calif, USA, 2000.

[25] Y. Guo, D. K. Kwon, and A. Kareem, "Near-real-time hybrid system identification framework for civil structures with application to Burj Khalifa," Journal of Structural Engineering, vol. 142, no. 2, Article ID 04015132, 2016.

[26] F. Auger and P. Flandrin, "Improving the readability of timefrequency and time-scale representations by the reassignment method," IEEE Transactions on Signal Processing, vol. 43, no. 5, pp. 1068-1089, 1995.

[27] P. Flandrin, F. Auger, and E. Chassande-Mottin, "Time-frequency reassignment: from principles to algorithms," in Applications in Time-Frequency Signal Processing, A. PapandreouSuppappola, Ed., 2003.

[28] F. Auger, P. Flandrin, Y.-T. Lin et al., "Time-frequency reassignment and synchrosqueezing: an overview," IEEE Signal Processing Magazine, vol. 30, no. 6, pp. 32-41, 2013.

[29] Z. Peng, F. Chu, and Y. He, "Vibration signal analysis and feature extraction based on reassigned wavelet scalogram," Journal of Sound and Vibration, vol. 253, no. 5, pp. 1087-1100, 2003.

[30] M. M. Khatibi, M. R. Ashory, A. Malekjafarian, and R. Brincker, "Mass-stiffness change method for scaling of operational mode shapes," Mechanical Systems and Signal Processing, vol. 26, no. 1, pp. 34-59, 2012.

[31] E. Parloo, P. Verboven, P. Guillaume, and M. Van Overmeire, "Sensitivity-based operational mode shape normalisation," Mechanical Systems and Signal Processing, vol. 16, no. 5, pp. 757-767, 2002.

[32] J.-N. Juang and R. S. Pappa, "An eigensystem realization algorithm for modal parameter identification and model reduction," Journal of Guidance, Control, and Dynamics, vol. 8, no. 5, pp. 620-627, 1985.

[33] M. H. Richardson, Modal Mass, Stiffness and Damping, Vibrant Technology, Inc., Jamestown, Calif, USA, 2000.

[34] M. S. Cao, G. G. Sha, Y. F. Gao, and W. Ostachowicz, "Structural damage identification using damping: a compendium of uses and features," Smart Materials and Structures, vol. 26, no. 4, Article ID 043001, 2017.

[35] G. H. James, T. G. Carne, and J. P. Lauffer, The Natural Excitation Technique (NExT) for modal parameter extraction from operating wind turbines, Sandia National Laboratories, 1993.

[36] S. R. Ibrahim, "Random decrement technique for modal identification of structures," Journal of Spacecraft and Rockets, vol. 14, no. 11, pp. 696-700, 1977.

[37] J. Luo, G. Liu, and Z.-M. Huang, "Modal parametric identification under non-stationary excitation based on random 
decrement method," Journal of Vibration and Shock, vol. 34, no. 21, pp. 19-24, 2015.

[38] P. Pozzati and C. Ceccoli, Teoria e tecnica delle strutture, vol. 2, UTET, 1988.

[39] G. Bernagozzi, L. Landi, and P. P. Diotallevi, “Truncation error analysis on modal flexibility-based deflections: application to mass regular and irregular structures," Engineering Structures, vol. 142, pp. 192-210, 2017.

[40] A. Saisi, C. Gentile, and M. Guidobaldi, "Post-earthquake continuous dynamic monitoring of the Gabbia Tower in Mantua, Italy," Construction and Building Materials, vol. 81, pp. 101-112, 2015.

[41] A. Cabboi, C. Gentile, and A. Saisi, "From continuous vibration monitoring to FEM-based damage assessment: application on a stone-masonry tower," Construction and Building Materials, vol. 156, pp. 252-265, 2017.

[42] F. Ubertini, G. Comanducci, and N. Cavalagli, "Vibrationbased structural health monitoring of a historic bell-tower using output-only measurements and multivariate statistical analysis," Structural Health and Monitoring, vol. 15, no. 4, pp. 438-457, 2016.

[43] F. Ubertini, G. Comanducci, N. Cavalagli, A. Laura Pisello, A. Luigi Materazzi, and F. Cotana, "Environmental effects on natural frequencies of the San Pietro bell tower in Perugia, Italy, and their removal for structural performance assessment," Mechanical Systems and Signal Processing, vol. 82, pp. 307-322, 2017.

[44] A. Deraemaeker, E. Reynders, G. De Roeck, and J. Kullaa, "Vibration-based structural health monitoring using outputonly measurements under changing environment," Mechanical Systems and Signal Processing, vol. 22, no. 1, pp. 34-56, 2008.

[45] J. Kullaa, "Structural health monitoring under nonlinear environmental or operational influences," Shock and Vibration, vol. 2014, Article ID 863494, 9 pages, 2014. 


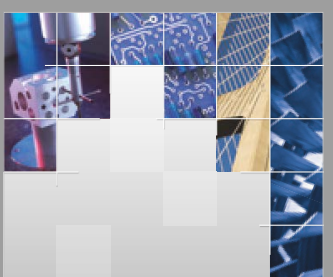

\section{Enfincering}
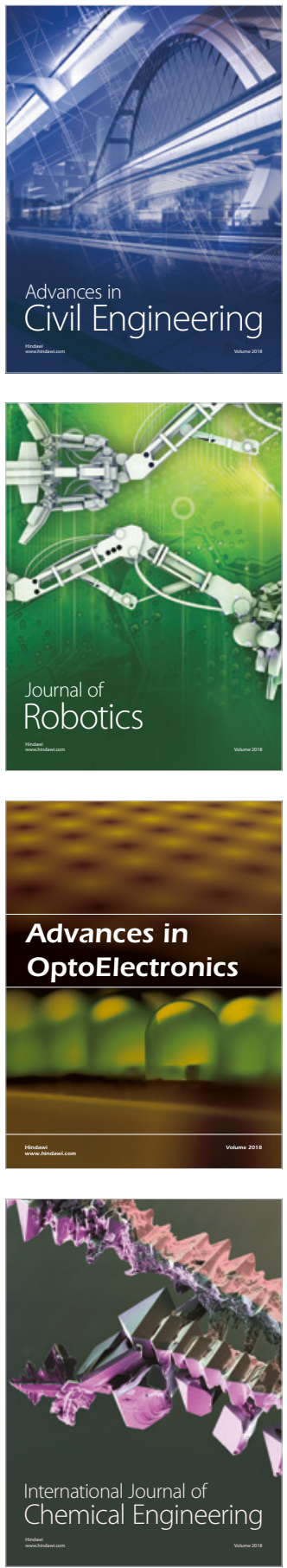

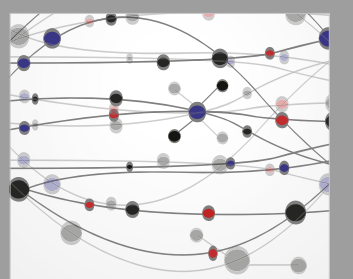

\section{Rotating \\ Machinery}

The Scientific World Journal

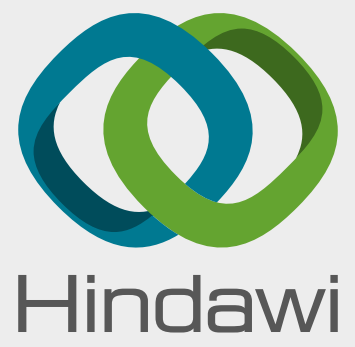

Submit your manuscripts at

www.hindawi.com
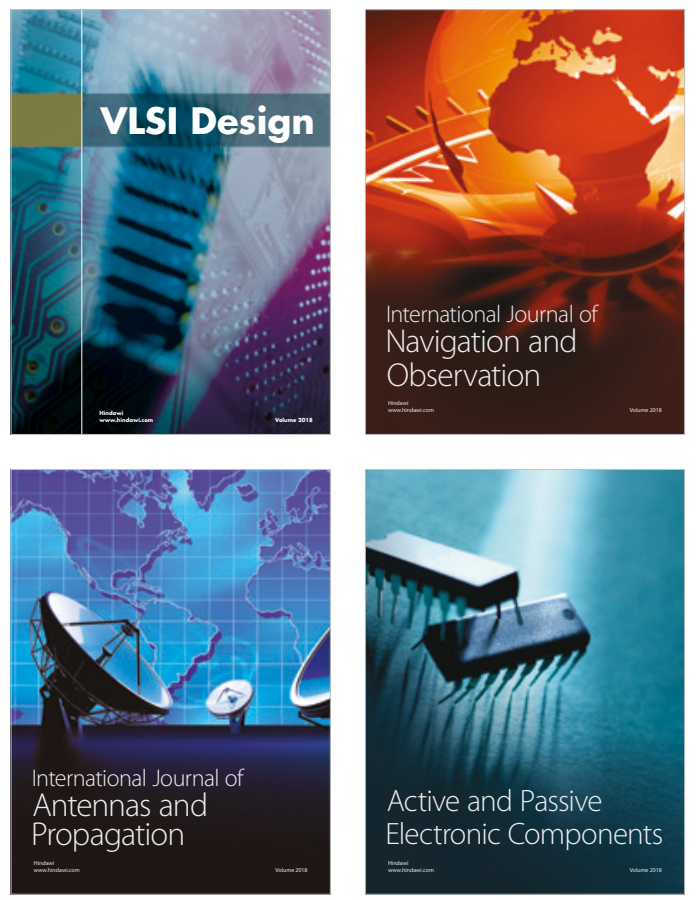
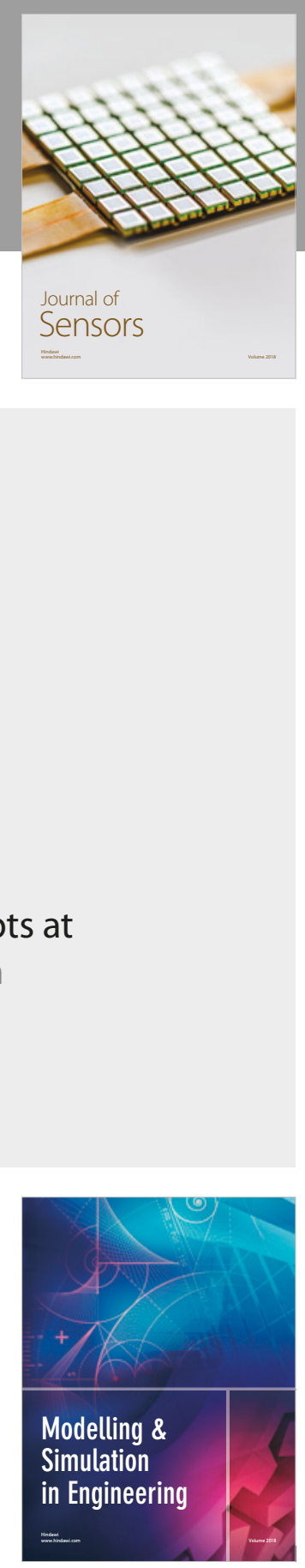

\section{Advances \\ Multimedia}
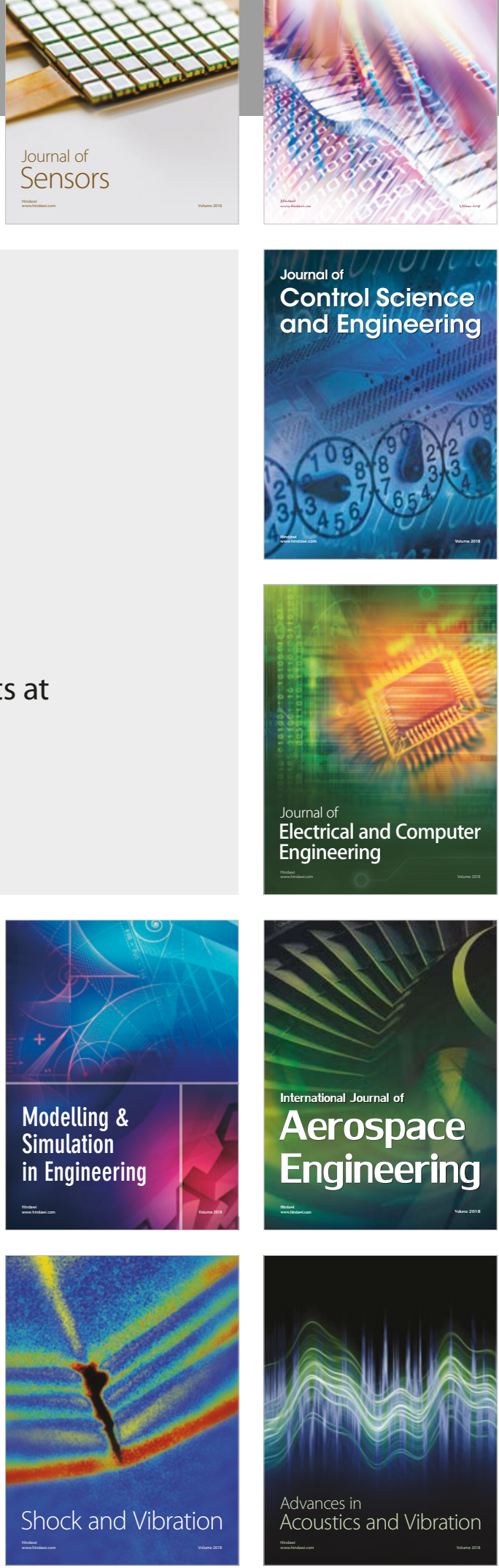